\title{
ON TWISTED GELFAND PAIRS THROUGH COMMUTATIVITY OF A HECKE ALGEBRA
}

\author{
YOTAM I. HENDEL
}

\begin{abstract}
For a locally compact, totally disconnected group $G$, a subgroup $H$ and a character $\chi: H \rightarrow \mathbb{C}^{\times}$we define a Hecke algebra $\mathcal{H}_{\chi}$ and explore the connection between commutativity of $\mathcal{H}_{\chi}$ and the $\chi$-Gelfand property of $(G, H)$, i.e. the property $\operatorname{dim}_{\mathbb{C}}\left(\rho^{*}\right)^{\left(H, \chi^{-1}\right)} \leq 1$ for every $\rho \in \operatorname{Irr}(G)$, the irreducible representations of $G$.

We show that the conditions of the Gelfand-Kazhdan criterion imply commutativity of $\mathcal{H}_{\chi}$, and verify in several simple cases that commutativity of $\mathcal{H}_{\chi}$ is equivalent to the $\chi$-Gelfand property of $(G, H)$.

We then show that if $G$ is a connected reductive group over a $p$-adic field $F$, and $G / H$ is $F$-spherical, then the cuspidal part of $\mathcal{H}_{\chi}$ is commutative if and only if $(G, H)$ satisfies the $\chi$-Gelfand property with respect to all cuspidal representations $\rho \in \operatorname{Irr}(G)$.

We conclude by showing that if $(G, H)$ satisfies the $\chi$-Gelfand property with respect to all irreducible $\left(H \backslash G, \chi^{-1}\right)$-tempered representations of $G$ then $\mathcal{H}_{\chi}$ is commutative.
\end{abstract}

\section{Contents}

1. Introduction

1.1. Motivation

1.2. Summary of the main results

1.3. Structure of the paper

1.4. Related work

1.5. Acknowledgments

1.6. Conventions and notations

2. Preliminaries

2.1. General representation theoretic facts and the Gelfand-Kazhdan criterion

2.2. The Bernstein decomposition

2.3. Cuspidal blocks

2.4. Direct integral decompositions of unitary representations

3. Definition of $\mathcal{H}_{\chi}$ and Gelfand-Kazhdan conditions imply $\mathcal{H}_{\chi}$ is commutative

4. $\mathcal{H}_{\chi}$ commutative implies $(G, H)$ is a $\chi$-Gelfand pair - geometric cases

4.1. Hecke pairs and Schlichting completions

5. $\mathcal{H}_{\chi}$ is commutative implies $(G, H)$ is a cuspidal $\chi$-Gelfand pair

6. A tempered $\chi$-Gelfand property implies $\mathcal{H}_{\chi}$ is commutative

Appendix A. A general proof of the Gelfand-Kazhdan criterion

References 


\section{INTRODUCTION}

\subsection{Motivation.}

1.1.1. Gelfand pairs of finite groups. Let $G$ be a finite group, and let $\operatorname{Irr}(G)$ denote the set of its irreducible representations. It is a fundamental result that the group algebra $\mathbb{C}[G]$ is semi-simple, and that each $\rho \in \operatorname{Irr}(G)$ appears $\operatorname{dim}_{\mathbb{C}} \rho$ times in its direct sum decomposition.

A question that comes to mind is what happens in the relative situation, i.e. given a subgroup $H \leq G$, how does the representation of $H$-invariant functions $\mathbb{C}[G]^{H} \simeq \mathbb{C}[H \backslash G]$ decompose. Here, not all irreducible representations of $G$ appear in $\mathbb{C}[H \backslash G]$, and those that do are called $H$-distinguished.

Definition 1.1. We say that $(G, H)$ is a Gelfand pair if every $\rho \in \operatorname{Irr}(G)$ appears in $\mathbb{C}[H \backslash G]$ at most once, i.e. $\operatorname{dim}_{\mathbb{C}} \operatorname{Hom}_{G}(\rho, \mathbb{C}[H \backslash G]) \leq 1$.

Note that by Frobenius reciprocity, this is equivalent to demanding that every $\rho \in \operatorname{Irr}(G)$ has at most one $H$-invariant linear functional up to a scalar $\left(\operatorname{dim}_{\mathbb{C}}\left(\rho^{*}\right)^{H}=\operatorname{dim}_{\mathbb{C}} \operatorname{Hom}_{H}\left(\left.\rho\right|_{H}, \mathbb{C}\right) \leq 1\right)$.

The notion of Gelfand pairs has found many uses throughout mathematics, and is used, for example, to study the representation theory of the symmetric group (e.g. OV96]) and when investigating the convergence rates of random walks on finite groups (e.g. Dia88, Chapter $3 \mathrm{~F}]$, [Let82]).

The Gelfand property can also be viewed as a relative analogue of Schur's lemma; indeed, in the group case, namely $\Delta G \leq G \times G$ where $\Delta G$ is a diagonal copy of $G$ embedded in $G \times G$, the Gelfand property of the pair $(G \times G, \Delta G)$ is a restatement of Schur's lemma.

In principle, in order to verify the Gelfand property of a pair $(G, H)$, one has to calculate the dimension of the space of $H$-invariant functionals on every $\rho \in \operatorname{Irr}(G)$, and show that in each case it is at most 1 . This is a hard task even in the case of finite groups, but conveniently the Gelfand property can be formulated in terms of commutativity of the convolution algebra of bi$H$-invariant functions on $G$, which in the case of finite groups is isomorphic to $\operatorname{End}_{G}(\mathbb{C}[H \backslash G])$. This simple observation is key to the work presented in this paper:

Observation 1.2. Let $H \leq G$ be finite groups. $(G, H)$ is a Gelfand pair $\Longleftrightarrow$ the Hecke algebra $\mathbb{C}[G]^{H \times H}$ is commutative.

Recall that an anti-involution $\sigma: G \rightarrow G$ is a map such that $\sigma\left(g_{1} g_{2}\right)=\sigma\left(g_{2}\right) \sigma\left(g_{1}\right)$ for all $g_{1}, g_{2} \in G$ and $\sigma^{2}=$ Id. In light of Observation 1.2, we can show that a pair has the Gelfand property using only the relative group structure of $G$ with respect to $H$, without knowing anything about the irreducible representations of $G$, or the decomposition of $\mathbb{C}[H \backslash G]$ :

Proposition 1.3 (Gelfand's trick). Assume there exists an anti-involution $\sigma$ of $G$ such that $\sigma(H)=H$ and $H \sigma(g) H=H g H$ for all $g \in G$, then $(G, H)$ is a Gelfand pair.

1.1.2. Non-compact subgroups and the Gelfand-Kazhdan criterion. The definition of a Gelfand pair can be generalized naturally to the case where $G$ is a locally compact group and $H \leq G$ is a compact subgroup, by demanding $\operatorname{dim}_{\mathbb{C}} \operatorname{Hom}_{H}\left(\left.\rho\right|_{H}, \mathbb{C}\right) \leq 1$ for every $\rho \in \operatorname{Irr}(G)$, where we either consider smooth irreducible representations of $G$ or unitary irreducible ones. In both these settings Observation 1.2 and Gelfand's trick hold (see vD09, Theorems 6.1.3 and 6.3.1], [BZ76, Section 2.10]), where $\mathbb{C}[G]^{H \times H}$ is replaced by $C_{c}(G)^{H \times H}$, the algebra of compactly supported, bi- $H$-invariant continuous functions on $G$. 
Let $G$ be a locally compact, totally disconnected topological group and let $H \leq G$ be a closed subgroup. We consider its category of smooth representations $\operatorname{Rep}(G)$. For simplicity, assume both $G$ and $H$ are unimodular. We would like to generalize the Gelfand property to these settings. While the straight-forward definition would be demanding $\operatorname{dim}_{\mathbb{C}} \operatorname{Hom}_{H}\left(\left.\rho\right|_{H}, \mathbb{C}\right) \leq 1$ for every $\rho \in \operatorname{Irr}(G)$ (GP1 as below), it is often much easier to prove an (a-priori) weaker statement (GP2 as below). There are no known examples of pairs $(G, H)$ which satisfy GP2 but not GP1, and it is conjectured that these two conditions are equivalent in general.

Definition 1.4 (In spirit of [AGS08, Definition 2.2.1]). Let $H \leq G$ be as above.

(1) We say that $(G, H)$ satisfies $G P 1$ if $\operatorname{dim}_{\mathbb{C}} \operatorname{Hom}_{H}\left(\left.\rho\right|_{H}, \mathbb{C}\right) \leq 1$ for every $\rho \in \operatorname{Irr}(G)$.

(2) We say that $(G, H)$ satisfies $G P 2$ if $\operatorname{dim}_{\mathbb{C}} \operatorname{Hom}_{H}\left(\left.\rho\right|_{H}, \mathbb{C}\right) \cdot \operatorname{dim}_{\mathbb{C}} \operatorname{Hom}_{H}\left(\left.\tilde{\rho}\right|_{H}, \mathbb{C}\right) \leq 1$ for every $\rho \in \operatorname{Irr}(G)$ and its smooth dual $\tilde{\rho}$.

Remark 1.5. The concept of Gelfand pairs in the case where $H$ non-compact have proven to be particularly important when studying periods in the theory of automorphic forms, see GPSR97, Gro91] or [Rez08].

Let $C^{-\infty}(G)$ denote the space of generalized functions on $G$, i.e. the dual of the space of all locally constant, compactly supported measures on $G$. The main mechanism used to show the Gelfand property (in the sense of GP2) in these settings is the following generalization of Gelfand's trick (see GK75], [Pra90, Lemma 4.2] or [Gro91, Proposition 4.2]).

Theorem 1.6 (Gelfand-Kazhdan criterion). Assume there exists an anti-involution $\sigma: G \rightarrow G$ such that $\sigma(H)=H$ and $\sigma(\xi)=\xi$ for every generalized function $\xi \in C^{-\infty}(G)^{H \times H}$, then $(G, H)$ is a Gelfand pair (in the sense of GP2).

When comparing the above to the case where $H$ is compact, we see that the space $C_{c}(G)^{H \times H}$ is replaced by the space of invariant generalized functions, which is not an algebra. Furthermore, it is not clear what is the analogue of Observation 1.2, if it exists. Evidently, if such an algebra existed, validity of the conditions of the Gelfand-Kazhdan criterion would imply it is commutative. We arrive at the following question.

Question 1.7. Can one define a Hecke algebra $\mathcal{H}$, analogous to $C_{c}(G)^{H \times H}$, such that

(1) The Gelfand-Kazhdan conditions imply commutativity of $\mathcal{H}$.

(2) $\mathcal{H}$ is commutative if and only if $(G, H)$ is a Gelfand pair.

1.2. Summary of the main results. Let $G$ be a locally compact, totally disconnected topological group, let $H \leq G$ be a closed subgroup, and let $\chi: H \rightarrow \mathbb{C}^{\times}$be a smooth character. For simplicity, assume $(G, H)$ is a unimodular pair. We say that $(G, H)$ is a $\chi$-Gelfand pair if GP1 holds where $\mathbb{C}$ is replaced by $\mathbb{C}_{\chi}$, i.e. $d_{H, \chi}(\rho):=\operatorname{dim}_{\mathbb{C}}\left(\rho^{*}\right)^{\left(H, \chi^{-1}\right)} \leq 1$ for all $\rho \in \operatorname{Irr}(G)$.

Let $\operatorname{ind}_{H}^{G}$ denote the compact induction functor. In this paper we define a Hecke algebra $\mathcal{H}_{\chi}:=$ $\operatorname{End}_{G}\left(\operatorname{ind}_{H}^{G} \chi^{-1}\right)$, which can conveniently be considered as an algebra of invariant distributions, and give a partial answer to Question 1.7 as follows. We first answer 1.7(1) affirmatively (set $\mathcal{H}:=\mathcal{H}_{1}$, where 1 is the trivial character of $\left.H\right)$ :

Theorem A (See Theorem 3.9 for a more general result). Assume the Gelfand-Kazhdan conditions hold (Proposition 1.6), i.e. there exists an anti-involution $\sigma: G \rightarrow G$ such that $\sigma(H)=H$ and $\sigma(\xi)=\xi$ for every $\xi \in C^{-\infty}(G)^{H \times H}$, then $\mathcal{H}$ is commutative. 
We then address [1.7(2) in several cases where $H \backslash G$ is especially well behaved:

Theorem B (For exact conditions on $\chi$ see Corollary 4.2, Proposition 4.3 and Proposition 4.11).

(1) If $H$ is either compact or co-compact, then $\mathcal{H}_{\chi}$ is commutative $\Longleftrightarrow(G, H)$ is a $\chi$ Gelfand pair.

(2) If $H$ is open and commensurated in $G$ (i.e. $g H g^{-1} \cap H$ has finite index in $H$ for all $g \in G)$, then $\mathcal{H}_{\chi}$ is commutative if and only if $d_{H, \chi}(\rho) \leq 1$ for every $\rho \in \operatorname{Irr}(G)$ such that either $\rho^{H_{S}} \neq\{0\}$ for a finite set $S \subset G / H$ where $H_{S}=H \bigcap_{g \in S} g H g^{-1}$, or $\rho$ is admissible.

In particular, we see that if $H$ is compact then $\mathcal{H}$ is commutative $\Longleftrightarrow C_{c}(G)^{H \times H}$ is commutative, so it is sensible to regard $\mathcal{H}$ as a generalization of $C_{c}(G)^{H \times H}$.

Furthermore, using Theorem $\mathrm{B}(2)$ we show that given a reductive algebraic group $\mathbf{G}$ satisfying the strong approximation property (see Theorem 4.15), the pair of discrete groups $(G, H)=$ $(\mathbf{G}(\mathbb{Q}), \mathbf{G}(\mathbb{Z}))$ satisfies $d_{H, 1}(\rho) \leq 1$ for every smooth irreducible representation $\rho$ of $G$ such that $\rho^{H_{S}} \neq\{0\}$ for some finite set $S \subset G / H$ and $H_{S}$ as above (note $\mathbf{G}(\mathbb{Z})$ is open and commensurated in $\mathbf{G}(\mathbb{Q})$ ). This is done by translating the Gelfand property of a classical pair to the settings of $(\mathbf{G}(\mathbb{Q}), \mathbf{G}(\mathbb{Z})$ ) via the Schlichting completion (see Section 4.1).

For the next result, assume $G=\mathbf{G}(F)$ and $H=\mathbf{H}(F)$, where $\mathbf{G}$ is a connected reductive group, $\mathbf{H} \leq \mathbf{G}$ a Zariski closed subgroup, and $F$ is a $p$-adic field. We also assume $(G, H)$ is an $F$-spherical pair (see Definition 2.13).

It is conjectured that $F$-sphericity implies $d_{H, \psi}(\rho)=\operatorname{dim}_{\mathbb{C}} \operatorname{Hom}_{G}\left(\operatorname{ind}_{H}^{G} \psi^{-1}, \tilde{\rho}\right)<\infty$ for every admissible $\rho \in \operatorname{Rep}(G)$ and character $\psi$ of $H$ (this was verified in several cases - see [Del10, [SV17]). In order to prove Theorem [C, it would be sufficient to assume $d_{H, \chi}(\rho)$ is finite for every irreducible cupsidal representation of $G$.

By analyzing $d_{H, \chi}(\rho)$ over the cuspidal blocks of $\operatorname{Rep}(G)$, and using [AS20, Theorem 6.1] and results from AAG12 we show the following:

Theorem $\mathbf{C}$ (See Theorem 5.5 for a more general result). The cuspidal part of $\mathcal{H}_{\chi}$ is commutative $\Longleftrightarrow(G, H)$ is a cuspidal $\chi$-Gelfand pair, i.e. $d_{H, \chi}(\rho) \leq 1$ for every cuspidal $\rho \in \operatorname{Irr}(G)$.

Remark 1.8. Note that for a cuspidal representation $\rho \in \operatorname{Irr}(G)$, we have $d_{H, \chi}(\rho)=d_{H, \chi^{-1}}(\tilde{\rho})$, so the conditions GP1 and GP2 (as in Definition 1.4) are equivalent when restricting to the class of irreducible cuspidal representations of $G$.

The last result of this paper concerns the converse direction of $1.7(2)$ :

Theorem D (See Theorem 6.2 for a more general result). Let $G$ be a second countable group of type I, and let $\chi$ be a unitary character. If $d_{H, \chi}(\rho) \leq 1$ for every $\left(H \backslash G, \chi^{-1}\right)$-tempered $\rho \in \operatorname{Irr}(G)$ (i.e. included in the support of the Plancherel measure of $L^{2}\left(H \backslash G, \chi^{-1}\right)$ ), then $\mathcal{H}_{\chi}$ is commutative. In particular, if $(G, H)$ is a $\chi$-Gelfand pair, then $\mathcal{H}_{\chi}$ is commutative.

1.3. Structure of the paper. The paper is structured as follows: In Section 2 we review facts from the representation theory of $p$-adic groups, the Gelfand-Kazhdan criterion, the Bernstein decomposition and the direct integral decomposition of unitary representations. In Section 3 we prove Theorem A. In Section 4 we prove Theorem B. In Section 5 we prove Theorem C. 
In Section 6 we prove Theorem D. In Appendix A we present a proof to a general version (i.e. twisted, non-unimodular) of the Gelfand-Kazhdan criterion.

1.4. Related work. The dimensions $d_{H, \chi}(\rho)$ (where $\rho$ ranges over $\operatorname{Irr}(G)$ ) and the Gelfand property have been studied vastly (e.g. Sha74, GK75, Pra90, Hak03, [AGS08, HM08, [Del10], [SZ11, AG09], AGRS10, AAG12], [SV17]) where in almost all cases proving that a pair is a Gelfand pair (where $H \leq G$ is non-compact) passes through the Gelfand-Kazhdan criterion.

The Gelfand-Kazhdan criterion was first introduced in [GK75, where it was used to show uniqueness of Whittaker models in the case of $\mathrm{GL}_{n}$ over a non-Archimedean local field.

In [SZ11], the autors give a general formulation of the Gelfand-Kazhdan criterion for real reductive Lie groups.

In Hak03] the author shows that for a $p$-adic symmetric pair $(G, H, \theta)$, it is enough to demand that $Z H \theta(g) H=Z H g^{-1} H$ for almost every double coset (i.e. $\left\{g \in G: Z H \theta(g) H \neq Z H g^{-1} H\right\}$ has measure zero in $G$ ) in order for $(G, H)$ to be a cuspidal Gelfand pair. It will be interesting to see whether this condition implies commutativity of our $\mathcal{H}_{\chi}$.

1.5. Acknowledgments. I wish to thank Uri Bader, Roman Bezrukavnikov, Shachar Carmeli, Max Gurevich, Dmitry Gourevitch, Erez Lapid, Omer Offen, Eitan Sayag and Michael Schein for various helpful discussions. Special thanks are due to Gil Goffer and Waltraud Lederle for their help with Section 4.1 and to Itay Glazer for numerous helpful discussions and for reading a preliminary version of this paper. I also wish to thank my advisors Avraham Aizenbud and Joseph Bernstein for their help and guidance throughout this project. Lastly, I wish to thank the anonymous referee for their time and effort, and for their valuable suggestions and remarks.

This work has particularly benefited from my participation in the doctoral school "Introduction to Relative Aspects in Representation Theory, Langlands Functoriality and Automorphic Forms" at CIRM, and in the "Sphericity 2016" conference. I wish to thank the organizers of both conferences.

This work was partially supported by ISF grants 687/13 and 249/17, BSF grant 2012247, ERC StG grant 637912 and by a Minerva foundation grant.

1.6. Conventions and notations. Throughout this paper $F$ is a non-Archimedean local field of characteristic zero. Boldface letters denote algebraic groups (such as $\mathbf{G}, \mathbf{P}$ ), and the corresponding non-boldface letters denote their $F$-points. Unless stated otherwise, $G$ is assumed to be a locally compact, totally disconnected (abbreviated l.c.t.d), unimodular, Hausdorff topological group, and $H$ is assumed to be a closed subgroup, not necessarily unimodular. $\chi: H \rightarrow \mathbb{C}^{\times}$ is assumed to a character of $H$.

We write $\delta:=\left(\delta_{G} \delta_{H}^{-1}\right)^{\frac{1}{2}}$ where $\delta_{G}$ and $\delta_{H}$ are the modular characters of $G$ and $H$ respectively, and $\tilde{\rho}:=\left(\rho^{*}\right)^{\mathrm{sm}}$ for the smooth dual of a representation $\rho$ of $G$. We also use $\rho^{H, \chi}:=\{v \in \rho$ : $h \cdot v=\chi(h) v \forall h \in H\}$.

We write $C_{c}^{\infty}(X)$ for the space of smooth (i.e. uniformly locally constant), compactly supported functions on $X$ and $\operatorname{Dist}(X)$ for the space of distributions on $X$, i.e. all linear functionals on $C_{c}^{\infty}(X)$. For a function $f$ on $G$ and a map $\sigma: G \rightarrow G$ we write $f^{\sigma}=f \circ \sigma$, and use $\left\langle\xi^{\sigma}, f\right\rangle=\left\langle\xi, f^{\sigma}\right\rangle$ for distributions. 


\section{PReliminaries}

2.1. General representation theoretic facts and the Gelfand-Kazhdan criterion. Recall we consider the category $\operatorname{Rep}(G)$ of smooth representation of a group $G$, where $\operatorname{Irr}(G)$ denotes the set of its irreducible objects (up to isomorphism). Let $\pi$ be a representation of $H$ and let $\chi: H \rightarrow \mathbb{C}^{\times}$be a character of $H$.

Definition 2.1. We define the induction and compact induction functors as follows.

(1) $\operatorname{Ind}_{H}^{G} \pi:=\{f: G \rightarrow \mathbb{C}: f(h g)=\pi(h)(f(g))$ and $f$ is smooth $\}$ where the action is $g^{\prime} \cdot f(g)=f\left(g g^{\prime}\right)$.

(2) $\operatorname{ind}_{H}^{G} \pi:=\left\{f \in \operatorname{Ind}_{H}^{G} \pi: \operatorname{supp}(f)\right.$ is compact modulo $\left.H\right\}$.

Lemmas 2.2 and 2.3 are standard, and are used extensively throughout the paper.

Lemma 2.2. Let $\rho$ be an irreducible representation of $G$.

(1) $\widetilde{\operatorname{ind}_{H}^{G} \pi} \simeq \operatorname{Ind}_{H}^{G}\left(\widetilde{\pi} \otimes \delta_{H \backslash G}\right)$.

(2) $\operatorname{Hom}_{G}\left(\operatorname{ind}_{H}^{G} \chi^{-1} \delta, \rho\right) \simeq \operatorname{Hom}_{G}\left(\tilde{\rho}, \operatorname{Ind}_{H}^{G} \delta \chi\right)$ if $\rho$ is admissible.

(3) $\operatorname{Hom}_{G}\left(\rho, \operatorname{Ind}_{H}^{G} \pi\right) \simeq \operatorname{Hom}_{H}\left(\rho_{\mid H}, \pi\right)$.

(4) $\operatorname{Hom}_{G}\left(\operatorname{ind}_{H}^{G} \pi, \rho\right) \simeq \operatorname{Hom}_{H}\left(\pi, \rho_{\mid H}\right)$ if $H$ is open.

Lemma 2.3. $\left(\operatorname{ind}_{H}^{G} \chi\right)^{*} \simeq \operatorname{Dist}(G)^{H, \chi \delta_{H}}$.

Proof. Define a map $\Phi: C_{c}^{\infty}(G) \rightarrow \operatorname{ind}_{H}^{G} \chi$ by $\Phi_{f, \chi}(g)=\int_{H} f(h g) \chi^{-1}(h) d \nu_{H}$. The image satisfies the necessary equivariance conditions, and its dual gives the desired isomorphism (see Off11, Lemma 3.1]).

Set $d_{H, \chi}(\rho):=\operatorname{dim}_{\mathbb{C}} \operatorname{Hom}_{H}\left(\left.\rho\right|_{H}, \chi\right)$. The following generalizes Definition 1.4(1) to nonunimodular pairs.

Definition 2.4. We say that $(G, H)$ is a $\chi$-Gelfand pair if $\operatorname{dim}_{\mathbb{C}} \operatorname{Hom}_{H}\left(\left.\rho\right|_{H}, \chi \delta\right) \leq 1$ for every irreducible representation $\rho$ of $G$.

Recall that an involution $\mu: G \rightarrow G$ is an automorphism of $G$ of order 2, and that an antiinvolution $\sigma$ is a map of the form $\sigma(g)=\mu(g)^{-1}$ where $\mu$ is an involution. The following is immediate.

Lemma 2.5. Let $\mu$ be an involution of $G$, and let $\nu_{G}$ be a left invariant Haar measure on $G$. Then changing variables $x \mapsto \mu(x)$ preserves $\nu_{G}$, that is $\mu_{*}\left(\nu_{G}\right)=\nu_{G}$.

We now state a general version of the Gelfand-Kazhdan criterion for future reference (for a proof see Appendix (A).

Theorem 2.6. (Gelfand-Kazhdan criterion) Assume there exists an anti-involution $\sigma: G \rightarrow G$ such that $\sigma(H)=H$ and such that for every $\xi \in C^{-\infty}(G)^{\left(H, \chi^{-1}\right) \times\left(H, \chi^{\sigma}\right)}$, i.e. $\xi$ where

$$
L_{h}(\xi)=\chi^{-1}(h) \xi, R_{h}(\xi)=\chi^{\sigma}(h) \xi
$$

we have $\xi^{\sigma}=\xi$. Then $d_{H, \chi}(\rho) \cdot d_{H, \chi^{\mu}}(\tilde{\rho}) \leq 1$ for every $\rho \in \operatorname{Irr}(G)$, where $\mu(g)=\sigma(g)^{-1}$. 
2.2. The Bernstein decomposition. Let $\mathbf{G}$ be a reductive algebraic group and set $G=$ $\mathbf{G}(F)$. The theory of the Bernstein center allows us to study $\operatorname{Rep}(G)$ by decomposing it into smaller indecomposable sub-categories called Bernstein blocks. We give a short review of the parts of this theory which are used in this work, see [BD84, [BR], or [Ro09] for a thorough treatment. We first need to establish some notations.

Definition 2.7. Let $G$ be as above. We define $G_{0} \leq G$ to be the inverse image of the maximal compact subgroup of $G /[G, G]$. Alternatively, this is the subgroup of $G$ generated by all compact subgroups.

The idea is that the representation theories of $G$ and $G_{0}$ are closely related, while $G_{0}$ is simpler (i.e. it has compact center). We get that $G_{0}$ is an open normal subgroup, and that $G / G_{0}$ is a finitely generated, discrete, abelian group (see [BR, Proposition 22]). Evidently, this gives the set of characters $\operatorname{Hom}_{G}\left(G / G_{0}, \mathbb{C}^{\times}\right)$a structure of an algebraic torus $\left(\mathbb{C}^{\times}\right)^{l}$ where $l$ is the rank of $G / G_{0}$.

Definition 2.8. We denote the variety $\operatorname{Hom}_{G}\left(G / G_{0}, \mathbb{C}^{\times}\right)$by $\mathfrak{X}_{G}$, and call a character $\chi \in \mathfrak{X}_{G}$ unramified.

Recall that a representation of $G$ is cuspidal if the support of its matrix coefficients is compact when projected to $G / Z(G)$ where $Z(G)$ is the center of $G$. A cuspidal datum of $G$ is a pair $(M, \rho)$ where $M=\mathbf{M}(F)$ is a Levi of a parabolic subgroup of $\mathbf{G}$ and $\rho$ an irreducible cuspidal representation of $M$. We define an equivalence relation on the set of cuspidal data by $(M, \rho) \sim$ $\left(M^{\prime}, \rho^{\prime}\right)$ if there exists $g \in G$ such that the following holds, where $\operatorname{Int}(g)$ is the conjugation action:

(1) $\operatorname{Int}(g)(M)=M^{\prime}$;

(2) $\operatorname{Int}(g)(\rho) \simeq \rho^{\prime} \otimes \chi$, where $\chi \in \mathfrak{X}_{M}$.

We denote the equivalence class of $(M, \rho)$ by $[M, \rho]$. We can now describe the Bernstein blocks:

Definition 2.9. Let $(M, \rho)$ be a cuspidal datum.

(1) We set $\Psi(M, \rho)=i_{G M}\left(\operatorname{ind}_{M_{0}}^{M}\left(\left.\rho\right|_{M_{0}}\right)\right)$ where $i_{G M}$ is the normalized parabolic induction from $M$ to $G$.

(2) The block $\mathcal{B}_{(M, \rho)}$ is defined to be the full subcategory of $\operatorname{Rep}(G)$ generated by $\Psi(M, \rho)$.

(3) Let $R_{(M, \rho)}:=\operatorname{End}_{G}(\Psi(M, \rho))$, and define the functor $H_{(-)}^{(M, \rho)}: \operatorname{Rep}(G) \rightarrow \operatorname{Mod}\left(R_{(M, \rho)}\right)$ by $H_{V}^{(M, \rho)}=\operatorname{Hom}_{G}(\Psi(M, \rho), V)$.

Proposition 2.10. Let $(M, \rho)$ and $\left(M^{\prime}, \rho^{\prime}\right)$ be cuspidal data.

(1) The categories $\mathcal{B}_{(M, \rho)}$ and $\mathcal{B}_{\left(M^{\prime}, \rho^{\prime}\right)}$ are equal (as subcategories of $\operatorname{Rep}(G)$ ) if and only if $(M, \rho) \sim\left(M^{\prime}, \rho^{\prime}\right)$, so we may write $\mathcal{B}_{[M, \rho]}$ for the corresponding block.

(2) The functor $H_{(-)}^{(M, \rho)} \mathcal{B}_{(M, \rho)}: \mathcal{B}_{(M, \rho)} \rightarrow \operatorname{Mod}\left(R_{(M, \rho)}\right)$ is an equivalence of categories.

Proof. For (1) see [BR, Proposition 35]. For (2) see [BR, Lemma 22] and [BR, Theorem 23].

Let $\Omega$ be the set of equivalence classes of cuspidal data under $\sim$. The following theorem, due to Bernstein, describes the structure of $\operatorname{Rep}(G)$ :

Theorem 2.11. [BR, Decomposition Theorem] The category $\operatorname{Rep}(G)$ admits a decomposition $\operatorname{Rep}(G) \simeq \prod_{[M, \rho] \in \Omega} \mathcal{B}_{[M, \rho]}$. 
In particular, in many situations we can restrict our attention to one block at a time, which by Proposition 2.10 is equivalent to a category of modules. The blocks which will be important for us are the cuspidal blocks.

2.3. Cuspidal blocks. Fix an irreducible cuspidal representation $\rho$ of $G$, and set $\Psi(\rho):=$ $\Psi(G, \rho)=\operatorname{ind}_{G_{0}}^{G}\left(\left.\rho\right|_{G_{0}}\right)$, a projective generator of the block $\mathcal{B}_{[G, \rho]}$, and $H_{\rho,(-)}:=H_{(-)}^{(G, \rho)}$ for the projection to the block $\mathcal{B}_{[G, \rho]}$.

We have an isomorphism of algebras $B:=\left(\mathcal{O}\left(\mathfrak{X}_{G}\right), \cdot\right) \simeq\left(C_{c}\left[G / G_{0}\right], *\right)$ by choosing generators $G / G_{0} \simeq \mathbb{Z}^{l}$ and sending $f \in C_{c}\left[G / G_{0}\right]$ to $\sum_{i \in \mathbb{Z}^{l}} f(i) x^{i} \in \mathbb{C}\left[x_{1}^{ \pm 1}, \ldots, x_{l}^{ \pm 1}\right]$. Now, set $R_{\rho}=R_{(G, \rho)}$ and embed $B \simeq C_{c}\left[G / G_{0}\right]$ into $R_{\rho}$ by $(b \cdot f)(x)=b(x) f(x)$ for $f \in \Psi(\rho)$ and $b \in B$. Finally, set $Z_{\rho}=Z\left(R_{\rho}\right)$.

Theorem 2.12 ([ AS20, Proposition 4.5]). Let $Z_{\rho} \subseteq B \subseteq R_{\rho}$ be as above, then the following hold:

(1) After applying the functor $B \otimes_{Z_{\rho}}$ to the triple $Z_{\rho} \subseteq B \subseteq R_{\rho}$ we get

$$
B \cdot \operatorname{Id} \subseteq \operatorname{diag}(B) \subseteq \mathrm{M}_{n}(B),
$$

where $\mathrm{M}_{n}(B)$ denotes the algebra of $n \times n$ matrices with values in $B, \operatorname{diag}(B)$ its subalgebra of diagonal matrices and $B$. Id the subalgebra of scalar matrices with values in $B$.

(2) The map $\operatorname{Spec}(B) \rightarrow \operatorname{Spec}\left(Z_{\rho}\right)$ induced by the inclusion $Z_{\rho} \subseteq B$ is surjective and étale.

Next we define the property of being an $F$-spherical pair and state a result for such pairs, due to Aizenbud-Sayag, which is a main ingredient in the proof of Theorem C.

Definition 2.13. A pair $(G, H)$ is called $F$-spherical if $|H \backslash G / P|<\infty$ for every $P=\mathbf{P}(F)$ and $\mathbf{P}$ a parabolic of $\mathbf{G}$ defined over $F$.

Theorem 2.14 (Implications of [AS20, Theorems 4.3 and 6.1]). Assume $(G, H)$ is an Fspherical pair and that $d_{H, \chi}(\rho)<\infty$.

(1) $\operatorname{Hom}_{G}(V, \rho \otimes \psi) \simeq \operatorname{Hom}_{\mathcal{O}_{\mathfrak{x}_{G}}}\left(H_{\rho, V}, \delta_{\psi}\right)$ for every $V \in \operatorname{Rep}(G)$, where $\delta_{\psi}$ is the skyscraper sheaf over the trivial character $\psi \in \mathfrak{X}_{G}$ with ring $\mathcal{O}_{\mathfrak{X}_{G}, \psi} / \mathfrak{m}_{\psi}$, the residue field at $\psi$.

(2) The module $\left.H_{\rho, \operatorname{ind}_{H}^{G} \chi^{-1} \delta}\right|_{B}$ is locally free over a smooth subvariety of $\mathfrak{X}_{G}$.

2.4. Direct integral decompositions of unitary representations. We follow [KS18, Section 8] and [Füh05, Section 3.4], see these sources for a thorough treatment of direct integrals and disintegration of unitary representations.

Let $Z$ be a second countable topological space possessing a $\sigma$-finite Borel measure $\nu$.

Definition 2.15. Let $\alpha: S \rightarrow V=\int_{Z}^{\oplus} V_{z} d \nu(z)$ be a continuous morphism from a topological vector space $S$ to a Hilbert space $V$ given as a direct integral of the family $\left(V_{z}\right)_{z \in Z}$. We say that $\alpha: S \rightarrow V$ is pointwise defined if there exists a family of morphisms $\left\{\alpha_{z}: S \rightarrow V_{z}\right\}_{z \in Z}$ such that $\alpha(s)(z)=\alpha_{z}(s) \nu$-almost everywhere for every $s \in S$.

Assume $G$ is a second countable group of type I (see [Füh05, Definition 3.19]). The importance of this condition is that it implies the unitary representations of $G$ can be decomposed uniquely as a direct integral of representations over the unitary dual $\widehat{G}$ of $G$ (see [Füh05, Theorem 3.16] 
or [KS18, Section 8.2]). By a theorem of Bernstein, every reductive group over a non-discrete, non-Archimedean local field is of type I (Ber74]).

Given a unitary representation $\rho$ of $G$, it possesses a central decomposition as follows:

Theorem 2.16 ([Füh05, Theorem 3.24]). Let $G$ be a group of type I and let $(V, \rho)$ be a unitary representation of $G$. There exist a standard Borel measure $\nu_{\rho}$ on $\widehat{G}$ and a multiplicity function $m_{\rho}: \widehat{G} \rightarrow \mathbb{N}_{0} \cup\{\infty\}$ such that

$$
\rho \simeq \int_{\widehat{G}}^{\oplus} m_{\rho}(\pi) \pi d \nu_{\rho}(\pi)
$$

Let $\chi$ be a unitary character, and let $\nu_{W(\chi)}$ be the measure appearing in the central decomposition of $W(\chi):=L^{2}\left(H \backslash G, \chi^{-1} \delta\right)$ as in Theorem 2.16, We call $\nu_{W(\chi)}$ the Plancherel measure of $L^{2}\left(H \backslash G, \chi^{-1} \delta\right)$, and say that a representation $\rho \in \operatorname{Irr}(G)$ is $\left(H \backslash G, \chi^{-1}\right)$-tempered if $\rho \in \operatorname{supp}\left(\nu_{W(\chi)}\right)$. This is equivalent to the existence of a functional $\xi \in\left(\rho^{*}\right)^{\left(H, \chi^{-1}\right)}$ such that the matrix coefficient $m_{\xi, v}$ has certain good growth conditions for every $v \in \rho$ (see [Ber88, Page 666 , Condition $(* * *)])$. We finish this section with the next proposition, which is used to deduce the main result of Section 5 .

Proposition 2.17 (See Ber88, Section 2.3] for the unimodular case or Ber88, Sections 3.2, 3.4 and 3.7] for the general one). The natural embedding $\alpha: \operatorname{ind}_{H}^{G} \chi^{-1} \delta \rightarrow L^{2}\left(H \backslash G, \chi^{-1} \delta\right)$ is pointwise defined.

\section{Definition of $\mathcal{H}_{\chi}$ and Gelfand-Kazhdan conditions imply $\mathcal{H}_{\chi}$ is commutative}

Let $H \leq G$ be l.c.t.d groups as in Section 1.6. Since $G$ is unimodular, $\delta_{G}=1$, and we have $\delta^{2}=\delta_{H \backslash G}=\delta_{H}^{-1}$.

Definition 3.1. We define the Hecke algebra to be $\mathcal{H}_{\chi}:=\operatorname{End}_{G}\left(\operatorname{ind}_{H}^{G} \chi^{-1} \delta\right)$.

Remark 3.2. If $G$ and $H$ are finite then $\mathcal{H}_{\chi} \simeq \mathbb{C}[G]^{(H, \chi) \times\left(H, \chi^{-1}\right)}$, so commutativity of $\mathcal{H}_{\chi}$ is equivalent to $(G, H)$ being a $\chi$-Gelfand pair (Definition 2.4). This shows $\mathcal{H}_{\chi}$ is a good candidate to generalize the usual Hecke algebra $\mathbb{C}[H \backslash G / H]$. In the next section we will see this also holds in the case where $H$ is compact and $G$ arbitrary.

While the Gelfand-Kazhdan criterion is usually phrased in terms of generalized functions, for us it will be easier to use distributions. Since $G$ is unimodular, choosing a Haar measure gives a (non-canonical) identification between these spaces.

Lemma 3.3. $C^{-\infty}(G) \simeq \operatorname{Dist}(G)$.

We wish to give $\mathcal{H}_{\chi}$ a geometric description as a space of invariant distributions. Recall $\left(\operatorname{ind}_{H}^{G} \chi\right)^{*} \simeq \operatorname{Dist}(G)^{\left(H, \chi \delta_{H}\right)}$ by Lemma 2.3. This gives rise to an isomorphism

$$
\left(\left(\operatorname{ind}_{H}^{G}\left(\chi^{-1} \delta\right) \otimes \operatorname{ind}_{H}^{G}(\chi \delta)\right)^{*}\right)^{\Delta G} \simeq \operatorname{Dist}(G \times G)^{\left(H, \chi^{-1} \delta^{-1}\right) \times\left(H, \chi \delta^{-1}\right) \times \Delta G} .
$$

Consider the map $i_{\chi}: \mathcal{H}_{\chi} \rightarrow\left(\left(\operatorname{ind}_{H}^{G}\left(\chi^{-1} \delta\right) \otimes \operatorname{ind}_{H}^{G}(\chi \delta)\right)^{*}\right)^{\Delta G}$ defined by

$$
\left\langle i_{\chi}(\tau), f_{1} \otimes f_{2}\right\rangle=\int_{H \backslash G} \tau\left(f_{1}(g)\right) f_{2}(g) d \mu_{H \backslash G} .
$$


Note that since $f_{1} \otimes f_{2} \in \operatorname{ind}_{H}^{G}\left(\chi^{-1} \delta\right) \otimes \operatorname{ind}_{H}^{G}(\chi \delta)$, we get $f_{1} \cdot f_{2} \in \operatorname{ind}_{H}^{G} \delta_{H \backslash G}$, on which we have a $G$-invariant functional ([BZ76, Theorem 1.21]). Furthermore, $\mathcal{H}_{\chi}$ is indeed embedded in the space above:

Lemma 3.4. The map $i_{\chi}$ is an injection.

Proof. Let $\tau \in \mathcal{H}_{\chi}$ be a non-zero element, and let $f_{1} \in \operatorname{ind}_{H}^{G} \chi^{-1} \delta$ be a function such that $\tau\left(f_{1}\right) \neq 0$. Take $e \neq g_{0} \in G$ such that $\tau\left(f_{1}\right)\left(g_{0}\right) \neq 0$, define $f_{2}\left(g_{0}\right)=\overline{\tau\left(f_{1}\right)\left(g_{0}\right)}$ and extend it via $f_{2}\left(h g_{0}\right)=\chi(h) \delta(h) f_{2}\left(g_{0}\right)$ for every $h \in H$. Now, take an open compact subgroup $K \subset G$ small enough such that $g_{0} K \cap H=\varnothing$ and such that $f_{1}\left(g_{0} K\right)=f_{1}\left(g_{0}\right)$, and set $f_{2}\left(g_{0} K\right)=f_{2}\left(g_{0}\right)$ and $f_{2}(x)=0$ if $x \notin H g_{0} K$ (if $H$ is open take $K=\{e\}$ ). Clearly, $f_{2} \in \operatorname{ind}_{H}^{G} \chi \delta$, and since $\left.\left(\tau\left(f_{1}\right) f_{2}\right)\right|_{H g_{0} K}$ is positive and vanishes outside $H g_{0} K$ we are done.

Lemma 3.5. We have,

$$
\left(\left(\operatorname{ind}_{H}^{G}\left(\chi^{-1} \delta\right) \otimes \operatorname{ind}_{H}^{G}(\chi \delta)\right)^{*}\right)^{\Delta G} \simeq \operatorname{Dist}(G)^{\left(H, \chi^{-1} \delta^{-1}\right) \times\left(H, \chi \delta^{-1}\right)} .
$$

Proof. To prove the lemma we apply Lemma 2.3 twice to show that both spaces are isomorphic to

$$
\operatorname{Dist}(G \times G)^{\left(H, \chi^{-1} \delta^{-1}\right) \times\left(H, \chi \delta^{-1}\right) \times \Delta G} .
$$

Assume the Gelfand-Kazhdan conditions with respect to $\chi \delta$ hold (see Theorem 2.6). This means there exists an anti-involution $\sigma$ of $G$ preserving $H$ such that $\xi^{\sigma}=\xi$ for all distributions $\xi$ belonging to the right hand side of $(*)$. Further assume that $\chi$ satisfies $\chi^{\sigma}=\chi$, and recall $\delta^{\mu}=\delta$ by Lemma 2.5, where $\mu(x)=\sigma\left(x^{-1}\right)$.

By the isomorphism above, $\sigma$ can be translated to an involution $\theta$ on the left hand side of $(*)$.

Proposition 3.6. Let $\varphi \in\left(\left(\operatorname{ind}_{H}^{G}\left(\chi^{-1} \delta\right) \otimes \operatorname{ind}_{H}^{G}(\chi \delta)\right)^{*}\right)^{\Delta G}$. The involution $\theta$ induced by $\sigma$ acts as follows:

$$
\left\langle\varphi^{\theta}, f_{1} \otimes f_{2}\right\rangle=\left\langle\varphi, f_{2}^{\mu} \otimes f_{1}^{\mu}\right\rangle
$$

We divide the proof into two lemmas, first passing through the involution $\tilde{\theta}$ induced on the space $\operatorname{Dist}(G \times G)^{\Delta G}$, and then showing that $\tilde{\theta}$ gives rise to the involution $\theta$ as above.

Lemma 3.7. The anti-involution $\sigma$ on $\operatorname{Dist}(G)$ induces the following on $\operatorname{Dist}(G \times G)^{\Delta G}$ :

$$
\left\langle\varphi^{\tilde{\theta}}, f_{1} \otimes f_{2}\right\rangle=\left\langle\varphi, f_{2}^{\mu} \otimes f_{1}^{\mu}\right\rangle .
$$

Proof. Consider the isomorphism $\Psi: \operatorname{Dist}(G) \simeq\left(\operatorname{ind}_{\Delta G}^{G \times G} 1\right)^{*} \stackrel{\sim}{\rightarrow} \operatorname{Dist}(G \times G)^{\Delta G}$ as in Lemma 2.3 ,

$$
\langle\Psi(\eta), f\rangle=\left\langle\eta, \Phi_{f}\right\rangle,
$$

where $\Phi_{f}(q)=\int_{G} f(q r, r) d r$ (here the $G \times G$-action on $\operatorname{ind}_{\Delta G}^{G \times G} 1$ is on the left). We have,

$$
\begin{gathered}
\left\langle\Psi\left(\eta^{\sigma}\right), f_{1} \otimes f_{2}\right\rangle=\left\langle\eta, \Phi_{f_{1} \otimes f_{2}}^{\sigma}\right\rangle, \\
\left\langle\Psi(\eta)^{\tilde{\theta}}, f_{1} \otimes f_{2}\right\rangle=\left\langle\Psi(\eta), f_{2}^{\mu} \otimes f_{1}^{\mu}\right\rangle=\left\langle\eta, \Phi_{f_{2}^{\mu} \otimes f_{1}^{\mu}}\right\rangle .
\end{gathered}
$$


Thus it is enough to show that $\Phi_{f_{2}^{\mu} \otimes f_{1}^{\mu}}=\Phi_{f_{1} \otimes f_{2}}^{\sigma}$. We calculate and get the following:

$$
\Phi_{f_{2}^{\mu} \otimes f_{1}^{\mu}}(q)=\int_{G} f_{2}^{\mu} \otimes f_{1}^{\mu}(q r, r) d r=\int_{G} f_{1} \otimes f_{2}(\mu(r), \mu(q r)) d r .
$$

Substitute $r^{\prime}=\mu(r)=\sigma\left(r^{-1}\right)$. Since $\mu$ is an involution, by Lemma 2.5 we have $\mu_{*} d r=d r^{\prime}$. We are now finished by the following:

$$
\int_{G} f_{1} \otimes f_{2}\left(r^{\prime}, \mu(q) r^{\prime}\right) d r^{\prime}=\int_{G} f_{1} \otimes f_{2}\left(\sigma(q) r^{\prime}, r^{\prime}\right) d r^{\prime}=\Phi_{f_{1} \otimes f_{2}}^{\sigma}(q) .
$$

Lemma 3.8. The involution $\tilde{\theta}$ on $\operatorname{Dist}(G \times G)^{\left(H, \chi^{-1} \delta^{-1}\right) \times\left(H, \chi \delta^{-1}\right) \times \Delta G}$ induces the involution $\theta$ on $\left(\left(\operatorname{ind}_{H}^{G}\left(\chi^{-1} \delta\right) \otimes \operatorname{ind}_{H}^{G}(\chi \delta)\right)^{*}\right)^{\Delta G}$.

Proof. As before have a map, $\Psi:\left(\left(\operatorname{ind}_{H}^{G}\left(\chi^{-1} \delta\right) \otimes \operatorname{ind}_{H}^{G}(\chi \delta)\right)^{*}\right)^{\Delta G} \rightarrow \operatorname{Dist}(G \times G)^{\left(H, \chi^{-1} \delta^{-1}\right) \times\left(H, \chi \delta^{-1}\right) \times \Delta G}$ by

$$
\left\langle\Psi(\xi), f_{1} \otimes f_{2}\right\rangle=\left\langle\xi, \Phi_{f_{1}, \chi^{-1} \delta} \otimes \Phi_{f_{2}, \chi \delta}\right\rangle
$$

where $\Phi_{f, \psi}(g)=\int_{H} f(h g) \psi^{-1}(h) d h$ as in Lemma 2.3 and $\psi \in\left\{\chi^{-1} \delta, \chi \delta\right\}$. Now, expanding,

$$
\left\langle\Psi(\xi)^{\tilde{\theta}}, f_{1} \otimes f_{2}\right\rangle=\left\langle\Psi(\xi), f_{2}^{\mu} \otimes f_{1}^{\mu}\right\rangle=\left\langle\xi, \Phi_{f_{2}^{\mu}, \chi^{-1} \delta} \otimes \Phi_{f_{1}^{\mu}, \chi \delta}\right\rangle .
$$

For every character $\psi$ of $H$ and $f \in C_{c}^{\infty}(G)$, it holds that,

$$
\Phi_{f^{\mu}, \psi}(x)=\int_{H} f(\mu(h) \mu(x)) \psi^{-1}(h) d h=\int_{H} f(h \mu(x)) \psi^{-1}(\mu(h)) d h=\Phi_{f, \psi^{\mu}}^{\mu},
$$

where $\mu_{*}(d h)=d h$ by Lemma 2.5. Back to our calculation, using the relations $\delta^{\mu}=\delta$ and $\chi^{\sigma}=\chi$ we conclude the desired statement:

$\left\langle\xi, \Phi_{f_{2}^{\mu}, \chi^{-1} \delta} \otimes \Phi_{f_{1}^{\mu}, \chi \delta}\right\rangle=\left\langle\xi, \Phi_{f_{2},\left(\chi^{-1} \delta\right)^{\mu}}^{\mu} \otimes \Phi_{f_{1},(\chi \delta)^{\mu}}^{\mu}\right\rangle=\left\langle\xi^{\theta}, \Phi_{f_{1}, \chi^{-1} \delta} \otimes \Phi_{f_{2}, \chi \delta}\right\rangle=\left\langle\Psi\left(\xi^{\theta}\right), f_{1} \otimes f_{2}\right\rangle$.

Using Proposition 3.6 we can now show the commutativity of $\mathcal{H}_{\chi}$. Recall we assume $\chi^{\sigma}=\chi$.

Theorem 3.9. Assume the conditions of the Gelfand-Kazhdan criterion hold with $\psi=\chi^{-1} \delta$ (Theorem [2.6), i.e. there exists an anti-involution $\sigma$ of $G$ such that $\sigma(H)=H$ and $\xi^{\sigma}=\xi$ for every $\xi \in \operatorname{Dist}(G)^{\left(H, \chi^{-1} \delta^{-1}\right) \times\left(H, \chi^{\sigma} \delta^{-1}\right)}$. Then $\mathcal{H}_{\chi}$ is commutative.

Proof. Take $\tau_{1}, \tau_{2} \in \mathcal{H}_{\chi}=\operatorname{End}_{G}\left(\operatorname{ind}_{H}^{G} \chi^{-1} \delta\right)$. Since $i_{\chi}$ is injective, it is enough to show that $i_{\chi}\left(\tau_{1} \circ \tau_{2}\right)=i_{\chi}\left(\tau_{2} \circ \tau_{1}\right)$, and since every $\xi$ in $\operatorname{Dist}(G)^{\left(H, \chi^{-1} \delta^{-1}\right) \times\left(H, \chi^{\sigma} \delta^{-1}\right)}$ is fixed under $\sigma$, for every $\tau \in \mathcal{H}_{\chi}$ we have $i_{\chi}(\tau)^{\theta}=i_{\chi}(\tau)$ where $\theta$ is as in Proposition 3.6. The statement is now reduced to the following calculation:

$$
\begin{aligned}
\left\langle i_{\chi}\left(\tau_{1} \circ \tau_{2}\right), f_{1} \otimes f_{2}\right\rangle & =\left\langle i_{\chi}\left(\tau_{1}\right), \tau_{2}\left(f_{1}\right) \otimes f_{2}\right\rangle=\left\langle i_{\chi}\left(\tau_{1}\right)^{\theta}, \tau_{2}\left(f_{1}\right) \otimes f_{2}\right\rangle \\
& =\left\langle i_{\chi}\left(\tau_{1}\right), f_{2}^{\mu} \otimes \tau_{2}\left(f_{1}\right)^{\mu}\right\rangle=\left\langle i_{\chi}(\mathrm{Id}), \tau_{1}\left(f_{2}^{\mu}\right) \otimes \tau_{2}\left(f_{1}\right)^{\mu}\right\rangle \\
& =\left\langle i_{\chi}(\mathrm{Id}), \tau_{2}\left(f_{1}\right) \otimes \tau_{1}\left(f_{2}^{\mu}\right)^{\mu}\right\rangle=\left\langle i_{\chi}\left(\tau_{2}\right), f_{1} \otimes \tau_{1}\left(f_{2}^{\mu}\right)^{\mu}\right\rangle \\
& =\left\langle i_{\chi}\left(\tau_{2}\right), \tau_{1}\left(f_{2}^{\mu}\right) \otimes f_{1}^{\mu}\right\rangle=\left\langle i_{\chi}\left(\tau_{2} \circ \tau_{1}\right), f_{2}^{\mu} \otimes f_{1}^{\mu}\right\rangle \\
& =\left\langle i_{\chi}\left(\tau_{2} \circ \tau_{1}\right), f_{1} \otimes f_{2}\right\rangle .
\end{aligned}
$$


4. $\mathcal{H}_{\chi}$ commutative implies $(G, H)$ is a $\chi$-Gelfand pair - Geometric cases

Let $\mathcal{H}_{\chi}:=\operatorname{End}_{G}\left(\operatorname{ind}_{H}^{G} \chi^{-1} \delta\right)$ be as in Definition 3.1 and recall $G$ is an 1.c.t.d unimodular group with a closed, not necessarily unimodular subgroup $H$. In this section we prove Theorem B. We start by showing that $\mathcal{H}_{\chi}$ generalizes the Hecke algebra from the classical case, that is for compact $H$ the pair $(G, H)$ is a $\chi$-Gelfand pair $\Longleftrightarrow \mathcal{H}_{\chi}$ is commutative.

Let $A(G)$ be the algebra of smooth, compactly supported measures on $G$, and recall that for compact $K$ the pair $(G, K)$ is a $\chi$-Gelfand pair if and only if $A(G)^{(K, \chi) \times\left(K, \chi^{-1}\right)}$ is commutative (see Section 1.1 .2 for $\chi=1$, general $\chi$ is similar).

Proposition 4.1. Assume $K:=H$ is compact. We have $A(G)^{(K, \chi) \times\left(K, \chi^{-1}\right)} \hookrightarrow \mathcal{H}_{\chi}$ as algebras. If furthermore $K$ is open, then this map is an isomorphism.

Proof. Let $\mu_{G}$ be a choice of a Haar measure on $G$. We have a map $\psi: A(G)^{(K, \chi) \times\left(K, \chi^{-1}\right)} \rightarrow \mathcal{H}_{\chi}$,

$$
\psi\left(f^{\prime} \mu_{G}\right)(f)(g)=\left(f^{\prime} \mu_{G} * f\right)(g)(x)=\int_{G} f(g) f^{\prime}\left(x g^{-1}\right) d \mu_{G} .
$$

It is a well defined $G$-morphism since $\psi\left(f^{\prime} \mu_{G}\right)(f) \in \operatorname{ind}_{K}^{G} \chi^{-1}$ :

$$
\psi\left(f^{\prime} \mu_{G}\right)(f)\left(h x g^{\prime}\right)=\int_{G} f(g) f^{\prime}\left(h x g^{\prime} g^{-1}\right) d \mu_{G}=\chi^{-1}(h) \psi\left(f^{\prime} \mu_{G}\right)\left(R_{g^{\prime}} f\right)(x) .
$$

It is injective since $\psi$ commutes with embedding of both spaces into $\operatorname{Dist}(G)^{(K, \chi) \times\left(K, \chi^{-1}\right)}$, which for $\mathcal{H}_{\chi}$ is given by Frobenius reciprocity. If $K$ is open, Lemma 2.2(4) shows $\psi$ is an isomorphism.

Corollary 4.2. Let $K:=H$ be a compact subgroup, and assume $G$ is second countable. Then $\mathcal{H}_{\chi}$ is commutative $\Longleftrightarrow(G, K)$ is a $\chi$-Gelfand pair.

Proof. If $\mathcal{H}_{\chi}$ is commutative, then by the previous lemma so is $A(G)^{(K, \chi) \times\left(K, \chi^{-1}\right)}$, implying that $(G, K)$ is a $\chi$-Gelfand pair.

The converse follows since by [BR, Separation Lemma], $\mathcal{H}_{\chi}$ embeds in $\prod_{\rho \in \operatorname{Irr}(G)} \operatorname{End}_{\mathbb{C}}\left(\operatorname{Hom}_{G}\left(\operatorname{ind}_{H}^{G} \chi^{-1}\right), \rho\right)$ which is commutative if $(G, H)$ is a Gelfand pair (see Theorem 6.2 for a proof assuming $G$ is of type I).

Proposition 4.3. Let $\chi$ be a unitary character of $H$, and assume that $H \backslash G$ is compact. Then $\mathcal{H}_{\chi}$ is commutative $\Longleftrightarrow(G, H)$ is a $\chi$-Gelfand.

Proof. Since $\chi$ is unitary, $\operatorname{ind}_{H}^{G} \chi^{-1} \delta$ is unitarizable, and by compactness of $H \backslash G$ the representation $\operatorname{Ind}_{H}^{G} \chi^{-1} \delta=\operatorname{ind}_{H}^{G} \chi^{-1} \delta$ is admissible. This implies it is semi-simple. Consequently, by Schur's lemma $\mathcal{H}_{\chi}=\operatorname{End}_{G}\left(\operatorname{ind}_{H}^{G} \chi^{-1} \delta\right)$ decomposes as a product of matrix algebras, $\prod_{\alpha \in I} \mathrm{M}_{n_{\alpha}}(\mathbb{C})$, where

$$
n_{\alpha}=\operatorname{dim}_{\mathbb{C}} \operatorname{Hom}_{G}\left(\operatorname{ind}_{H}^{G} \chi^{-1} \delta, \rho_{\alpha}\right),
$$

and $\left\{\rho_{\alpha}\right\}_{\alpha \in I}$ are the smooth irreducible representations of $G$ (up to equivalence). In particular $\mathcal{H}_{\chi}$ is commutative if and only if $n_{\alpha} \leq 1$ for every $\alpha \in I$. 
4.1. Hecke pairs and Schlichting completions. We now move to consider the case of Hecke pairs. These are pairs $(G, H)$ which behave as if $H$ was compact.

Definition 4.4. Let $G$ be a l.c.t.d group with a closed subgroup $H$.

(1) We say $H$ is commensurated in $G$ if $|H g H / H|<\infty$ for every $g \in G$.

(2) We say that $(G, H)$ is a Hecke pair if $H$ is open and commensurated in $G$.

Remark 4.5. While Hecke pairs are usually defined in the context of discrete groups, there exist interesting non-discrete Hecke pairs $(G, H)$ where $H$ is not compact, cocompact or normal (see the pair in Corollary 4.12).

Let $H \leq G$ be l.c.t.d groups as in Section 1.6. If $(G, H)$ is a Hecke pair, one can complete the group $G$ with respect to a certain topology arising naturally from $H$ and get a locally compact, totally disconnected, Hausdorff topological group $\hat{G}$ and a homomorphism $\beta: G \rightarrow \hat{G}$ such that $\hat{H}:=\overline{\beta(H)}$ is open and compact. The group $\hat{G}$ is usually called in the literature the Schlichting completion of $(G, H)$ (see KLQ08, Definitions 3.3, 3.4 and 3.7] or [RW, Definition 5.2]).

More explicitly, for a set $S \subset G / H$ define $H_{S}=H \cap \bigcap_{g \in S} g H g^{-1}$ and let $\mathcal{S}:=\{S \subset G / H$ : $S$ is finite $\}$. If $(G, H)$ is a Hecke pair and $S \in \mathcal{S}$, then $H_{S}$ has finite index in $H$. The collection $\left\{H_{S}: S \in \mathcal{S}\right\}$ forms a basis at identity for a group topology on $G$, and completing $G$ with respect to it yields the Schlichting completion of $(G, H)$.

Alternatively, note $\left\{G / H_{S}: S \in \mathcal{S}\right\}$ forms an inverse system. The Schlichting completion of $(G, H)$ can be described as follows:

Lemma 4.6 (KLQ08, Proposition 3.10]). $\hat{G} \simeq \underset{\underbrace{}_{S \in \mathcal{S}}}{\lim } G / H_{S}$.

Remark 4.7. Since we demand $\hat{G}$ is Hausdorff, we have $\operatorname{ker} \beta=\bigcap_{g \in G} g H g^{-1}$.

Let $\operatorname{Rep}(G)$ be the category of smooth representations of a group $G$, and for $H \leq G$ set

$$
\operatorname{Rep}_{H}(G):=\left\{V \in \operatorname{Rep}(G): V=\bigcup_{S \in \mathcal{S}} V^{H_{S}}\right\} .
$$

Note that an irreducible representation $\rho \in \operatorname{Rep}(G)$ lies in $\operatorname{Rep}_{H}(G)$ if $\rho^{H_{S}} \neq\{0\}$ for some $S \in \mathcal{S}$.

Theorem 4.8. Let $(G, H)$ be a Hecke pair. Then $\operatorname{Rep}_{H}(G) \cong \operatorname{Rep}(\hat{G})$.

Remark 4.9. Note that every $\rho \in \operatorname{Rep}_{H}(G)$ is trivial on the kernel of $\beta$ since $\operatorname{ker}(\beta) \subset H_{S}$ for every $S \in \mathcal{S}$. It follows that $\operatorname{Rep}_{H}(G) \cong \operatorname{Rep}_{H / N}(G / N)$ for $N=\operatorname{ker}(\beta)$, so it is enough to prove the theorem in the case where $\beta$ is injective.

Proof of Theorem 4.8. For $(V, \rho) \in \operatorname{Rep}_{H}(G)$ and $S \in \mathcal{S}$, set $\rho_{S}: G / H_{S} \rightarrow \operatorname{Hom}_{\mathbb{C}}\left(V^{H_{S}}, V\right)$ by $\rho_{S}\left(g H_{S}\right)(v)=\rho(g)(v)$. This is well defined since every $h_{S} \in H_{S}$ acts trivially on $V^{H_{S}}$. Now, by Lemma 4.6 we have maps $\hat{G} \rightarrow G / H_{S}$, and by precomposing we get maps $\hat{\rho}_{S}: \hat{G} \rightarrow$ $\operatorname{Hom}_{\mathbb{C}}\left(V^{H_{S}}, V\right)$.

Note that $\left\{\operatorname{Hom}_{\mathbb{C}}\left(V^{H_{S}}, V\right)\right\}_{S \in \mathcal{S}}$ forms an inverse system with respect to the natural restriction maps. These maps commute with the maps $\left\{\hat{\rho}_{S}\right\}_{S \in \mathcal{S}}$ defined above, and furthermore

$$
\underset{S \in \mathcal{S}}{\lim _{S}} \operatorname{Hom}_{\mathbb{C}}\left(V^{H_{S}}, V\right)=\operatorname{Hom}_{\mathbb{C}}\left(\underset{S \in \mathcal{S}}{\lim _{S}} V^{H_{S}}, V\right)=\operatorname{Hom}_{\mathbb{C}}(V, V) \text {. }
$$


By the universal property of $\underset{S \in \mathcal{S}}{\lim } \operatorname{Hom}_{\mathbb{C}}\left(V^{H_{S}}, V\right)$ we get a unique map $F(\rho):=\hat{\rho}: \hat{G} \rightarrow$ $\operatorname{End}_{\mathbb{C}}(V)$. By the construction $\left.\hat{\rho}\right|_{G}=\rho$; if $v \in V$ then $v \in V^{H_{S}}$ for some $S \in \mathcal{S}$, we get for every $\beta(g) \in \hat{G}$,

$$
\hat{\rho}(\beta(g))(v)=\hat{\rho}_{S}(\beta(g))(v)=\rho_{S}\left(g H_{S}\right)(v)=\rho(g)(v) .
$$

Note that $\hat{\rho}$ is continuous, i.e. for a given $v \in V$ the map $\hat{\rho}_{v}: g \mapsto \hat{\rho}(g)(v)$ is continuous. This implies that $\hat{\rho}$ is smooth, since for every $v \in V$ we have $\beta\left(H_{S}\right) \subset \operatorname{Stab}_{\hat{G}}(v)$ for some $S \in \mathcal{S}$, so $\overline{\beta\left(H_{S}\right)}$ stabilizes $v$. In particular, $\hat{\rho}$ is a homomorphism of groups to $\operatorname{GL}(V)$, since it is a homomorphism on a dense set $G \subset \hat{G}$, and similarly it follows that $F$ carries morphisms to morphisms. We conclude that $F((V, \rho))=(V, \hat{\rho}) \in \operatorname{Rep}(\hat{G})$.

Conversely, define $\operatorname{res}_{G}^{\hat{G}}: \operatorname{Rep}(\hat{G}) \rightarrow \operatorname{Rep}(G)$ by $\operatorname{res}_{G}^{\hat{G}}(\hat{\rho})(g)=\hat{\rho}(\beta(g))$, acting on the same space. Since every $\hat{V}$ is smooth and $\beta$ is continuous, every $\operatorname{res}_{G}^{\hat{G}}(\hat{V})$ is smooth. Furthermore, since a basis for the topology of $\hat{G}$ at identity is given by $\left\{\overline{\beta\left(H_{S}\right)}: S \in \mathcal{S}\right\}$, every $v \in \hat{V}$ is stabilized by some $\overline{\beta\left(H_{S}\right)}$, and thus every $v$ is stabilized by some $H_{S}$ as a representation of $G$.

Since by the construction $\operatorname{res}_{G}^{\hat{G}} \circ F=\operatorname{Id}_{\operatorname{Rep}_{H}(G)}$ and $F \circ \operatorname{res}_{G}^{\hat{G}}=\operatorname{Id}_{\operatorname{Rep}(\hat{G})}$, we are done.

Lemma 4.10. Let $(G, H)$ be a Hecke pair and let $\chi: H \rightarrow \mathbb{C}^{\times}$be a character such that $\chi\left(H_{S}\right)=1$ for some $S \in \mathcal{S}$. Then $\mathcal{H}_{\chi}(G, H) \simeq \mathcal{H}_{\hat{\chi}}(\hat{G}, \hat{H})$, where $\left.\hat{\chi}\right|_{H}=\chi$.

Proof. By the equivalence of categories, $\operatorname{End}_{G}\left(\operatorname{ind}_{H}^{G} \chi^{-1}\right) \simeq \operatorname{End}_{\hat{G}}\left(F\left(\operatorname{ind}_{H}^{G} \chi^{-1}\right)\right)$. Now $F\left(\operatorname{ind}_{H}^{G} \chi^{-1}\right)=$ $\operatorname{ind}_{\hat{H}}^{\hat{G}} \hat{\chi}^{-1}$, where $\hat{\chi}: \hat{H} \rightarrow \mathbb{C}^{\times}$is a character of $\hat{H}$ extending $\chi$. Note $H$ is open so $\delta=1$, and that an extension $\hat{\chi}$ exists since $H / \operatorname{ker} \chi \simeq \hat{H} / \operatorname{ker} \chi$ as finite groups.

Proposition 4.11. Let $(G, H)$ be a Hecke pair and $\chi: H \rightarrow \mathbb{C}^{\times}$as in Lemma 4.10. Then $\mathcal{H}_{\chi}$ is commutative $\Longleftrightarrow(G, H)$ satisfies the $\chi$-Gelfand property with respect to every irreducible representation in $\operatorname{Rep}_{H}(G)$. In particular, if $\mathcal{H}_{\chi}$ is commutative then $\operatorname{dim}_{\mathbb{C}} \operatorname{Hom}_{H}\left(\left.\rho\right|_{H}, \chi\right) \leq 1$ for every smooth irreducible admissible representation $\rho$ of $G$.

Proof. Let $M=\bigcup_{S \in \mathcal{S}}\left(\operatorname{Ind}_{H}^{G} \chi\right)^{H_{S}}$, then $F(M)=\operatorname{Ind}_{\hat{H}}^{\hat{G}} \hat{\chi}$ where $\hat{\chi}$ is a character of $\hat{H}$ extending $\chi$. Let $\rho \in \operatorname{Rep}_{H}(G)$ be an irreducible representation. By the equivalence of categories, we have

$$
\operatorname{dim}_{\mathbb{C}} \operatorname{Hom}_{G}\left(\rho, \operatorname{Ind}_{H}^{G} \chi\right)=\operatorname{dim}_{\mathbb{C}} \operatorname{Hom}_{G}(\rho, M)=\operatorname{dim}_{\mathbb{C}} \operatorname{Hom}_{\hat{G}}\left(F(\rho), \operatorname{Ind}_{\hat{H}}^{\hat{G}} \hat{\chi}\right),
$$

and in particular $(G, H)$ is a $\chi$-Gelfand pair with respect to $\operatorname{Rep}_{H}(G) \Longleftrightarrow(\hat{G}, \hat{H})$ is a $\hat{\chi}^{-}$ Gelfand pair. Using Lemma 4.10, we have $\mathcal{H}_{\chi}(G, H) \simeq \mathcal{H}_{\hat{\chi}}(\hat{G}, \hat{H})$, and since $\hat{H}$ is compact and open in $\hat{G}$, we get that $(\hat{G}, \hat{H})$ is a Gelfand pair $\Longleftrightarrow \mathcal{H}_{\hat{\chi}}(\hat{G}, \hat{H}) \simeq \mathcal{H}_{\chi}(G, H)$ is commutative.

If $\rho$ is an irreducible admissible representation of $G$ and $\operatorname{dim}\left(\rho^{*}\right)^{H}>0$, then $\tilde{\rho}$ is irreducible. Since $H$ is open $\operatorname{dim}\left(\rho^{*}\right)^{H}=\operatorname{dim}(\tilde{\rho})^{H}>0$ and we have $\tilde{\rho} \in \operatorname{Rep}_{H}(G)$. The claim now follows by the first part of the proof.

Corollary 4.12. Let $\mathcal{T}_{d}$ be a $d \geq 3$ regular tree, let $G \leq \operatorname{Aut}\left(\mathcal{T}_{d}\right)$ be the subgroup of automorphisms of $\mathcal{T}_{d}$ such that their local action is prescribed by a fixed finite permutation group $\{e\} \neq \Lambda<S_{d}$ around all but finitely many vertices, equipped with a l.c.t.d topology as in [LB16], and let $H$ be a stabilizer of a vertex $v$ in $\mathcal{T}_{d}$. Then $d_{H, 1}(\rho) \leq 1$ for every $\rho \in \operatorname{Irr}(G)$ which is either admissible or $\rho \in \operatorname{Rep}_{H}(G)$.

Proof. Since $G$ and $H$ are dense in $\operatorname{Aut}\left(\mathcal{T}_{d}\right)$ and $\operatorname{Stab}_{\text {Aut }\left(\mathcal{T}_{d}\right)}(v)$ respectively, where $\operatorname{Stab} \operatorname{Aut}\left(\mathcal{T}_{d}\right)(v)$ is open and compact, it follows that the Schlichting completion of $(G, H)$ is $\left(\operatorname{Aut}\left(\mathcal{T}_{d}\right), \operatorname{Stab}_{\mathrm{Aut}}\left(\mathcal{T}_{d}\right)(v)\right)$. 
The claim now follows from Lemma4.10 and Proposition 4.11, since the pair $\left(\operatorname{Aut}\left(\mathcal{T}_{d}\right), \operatorname{Stab}_{\mathrm{Aut}}\left(\mathcal{T}_{d}\right)(v)\right)$ is a Gelfand pair (see [Ol'77]).

Remark 4.13. Let $(G, H)$ and $\chi$ be as in Lemma 4.10 and let $\rho$ be an irreducible representation of $G$. If $\rho^{\left(H, \chi^{-1}\right)} \neq 0$ then $\rho \in \operatorname{Rep}_{H}(G)$. If $\rho$ is not admissible, it may happen that $\left(\rho^{*}\right)^{\left(H, \chi^{-1}\right)} \neq$ 0 but $\rho \notin \operatorname{Rep}_{H}(G)$, and in particular even if $\mathcal{H}_{\chi}$ is commutative we might have $\operatorname{dim}_{\mathbb{C}}\left(\rho^{*}\right)^{H}>1$.

Remark 4.14. If $H$ is open but $(G, H)$ is not a Hecke pair Proposition 4.11 fails. Let $T_{3}$ be the subgroup of diagonal matrices in $\mathrm{GL}_{3}\left(\mathbb{Q}_{p}\right)$, let $G:=N_{\mathrm{GL}_{3}\left(\mathbb{Q}_{p}\right)}\left(T_{3}\right)$ be its normalizer and set $H=\operatorname{diag}\left(\mathbb{Z}_{p}^{\times}, \mathbb{Q}_{p}^{\times}, \mathbb{Q}_{p}^{\times}\right) \leq T_{3}$. One can show $\mathcal{H} \simeq \mathbb{C}[\mathbb{Z} \times \mathbb{Z} / 2 \mathbb{Z}]$ is abelian, but the irreducible $G$-representation $V=\left\{\left(x_{1}, x_{2}, x_{3}\right) \in \mathbb{C}^{3}: \sum x_{i}=0\right\}$ with the $G$-action given by permuting the coordinates has $\operatorname{dim} V^{H}=2$. In particular $(G, H)$ is not a Gelfand pair.

4.1.1. Hecke pairs of algebraic groups. An algebraic group $\mathbf{G}$ defined over $\mathbb{Q}$ naturally gives rise to a Hecke pair by considering $(\mathbf{G}(\mathbb{Q}), \mathbf{G}(\mathbb{Z})$ ) (see PR94, Section 4.1, Corollary 1]). This has been famously used in BC95. Furthermore, in this case the Schlichting completion can be determined explicitly. One can then translate information from the relative representation theory of the completion to that of $(\mathbf{G}(\mathbb{Q}), \mathbf{G}(\mathbb{Z}))$.

Let $\mathbb{A}_{f}:=\prod_{p \text { prime }}^{\prime} \mathbb{Q}_{p}$ be the ring of finite adeles, and set $\hat{\mathbb{Z}}:=\prod_{p \text { prime }} \mathbb{Z}_{p}$. We will need the following.

Theorem 4.15 (Strong approximation, see [PR94, Theorem 7.12]). Let G be a connected, simply connected reductive algebraic group over $\mathbb{Q}$ with no $\mathbb{Q}$-simple components $\mathbf{G}^{i}$ where $\mathbf{G}^{i}(\mathbb{R})$ is compact. Then the diagonal embedding $\psi:(\mathbf{G}(\mathbb{Q}), \mathbf{G}(\mathbb{Z})) \hookrightarrow\left(\mathbf{G}\left(\mathbb{A}_{f}\right), \mathbf{G}(\hat{\mathbb{Z}})\right)$ is dense.

Assume $\mathbf{G}$ satisfies the conditions of the strong approximation theorem. Then $\psi$ is a completion map, and by the universal property of the Schlichting completion ([RW, Theorem 5.4]) the pair $\left(\mathbf{G}\left(\mathbb{A}_{f}\right), \mathbf{G}(\hat{\mathbb{Z}})\right)$ is the Schlichting completion of $(\mathbf{G}(\mathbb{Q}), \mathbf{G}(\mathbb{Z})$ ) (up to compact center). By Lemma 4.10, this implies

$$
\mathcal{H}(\mathbf{G}(\mathbb{Q}), \mathbf{G}(\mathbb{Z})) \simeq \mathcal{H}\left(\mathbf{G}\left(\mathbb{A}_{f}\right), \mathbf{G}(\hat{\mathbb{Z}})\right) \simeq \bigotimes^{\prime} \mathcal{H}\left(\mathbf{G}\left(\mathbb{Q}_{p}\right), \mathbf{G}\left(\mathbb{Z}_{p}\right)\right)=\bigotimes^{\prime} C_{c}^{\infty}\left(\mathbf{G}\left(\mathbb{Q}_{p}\right)\right)^{\mathbf{G}\left(\mathbb{Z}_{p}\right) \times \mathbf{G}\left(\mathbb{Z}_{p}\right)}
$$

We can thus restate the classical result that $\left(\mathbf{G}\left(\mathbb{A}_{f}\right), \mathbf{G}(\hat{\mathbb{Z}})\right)$ is a Gelfand pair in the language of discrete groups using Proposition 4.11. Note that in the case $\mathbf{G}=\mathrm{GL}_{2}$ the Hecke algebra $\mathcal{H}(\mathbf{G}(\mathbb{Q}), \mathbf{G}(\mathbb{Z}))$ recovers the classical algebra of Hecke operators on modular forms Cog04.

Corollary 4.16. Let $\mathbf{G}$ be a connected reductive algebraic group defined over $\mathbb{Q}$ satisfying the strong approximation property (Theorem 4.15). Then the following pairs $(G, H)$ satisfy $d_{H, 1}(\rho)=\operatorname{dim}_{\mathbb{C}} \operatorname{Hom}_{H}\left(\left.\rho\right|_{H}, \mathbb{C}\right) \leq 1$ for every irreducible representation $\rho \in \operatorname{Rep}_{H}(G)$ :

(1) $(G, H)=(\mathbf{G}(\mathbb{Q}), \mathbf{G}(\mathbb{Z}))$.

(2) $(G, H)=\left(\mathbf{G}\left(\mathbb{Z}\left[\frac{1}{p}\right]\right), \mathbf{G}(\mathbb{Z})\right)$.

(3) $(G, H)=\left(\mathbf{G}(\mathbb{Q}), \mathbf{G}\left(\mathbb{Z}\left[S_{p}\right]\right)\right)$, where $p$ is prime, $S_{p}=\left\{\frac{1}{p^{\prime}}: p^{\prime} \neq p\right.$ and $p^{\prime}$ is prime $\}$.

Remark 4.17. Item (1) of Corollary 4.16 above can be adapted to the case of a number field $K$.

\section{5. $\mathcal{H}_{\chi}$ is commutative implies $(G, H)$ is a Cuspidal $\chi$-Gelfand pair}

Let $\mathbf{G}$ be a connected reductive group with a Zariski closed subgroup $\mathbf{H} \leq \mathbf{G}$ and set $G=\mathbf{G}(F)$ and $H=\mathbf{H}(F)$. In this section we show that for $F$-spherical pairs (Definition 2.13) 
the cuspidal part of $\mathcal{H}_{\chi}$ is commutative if and only if $(G, H)$ is a cuspidal $\chi$-Gelfand pair, i.e. $\operatorname{dim}_{\mathbb{C}} \operatorname{Hom}_{H}\left(\left.\rho\right|_{H}, \chi \delta\right) \leq 1$ for every $\rho \in \operatorname{Irr}(G)$.

Since Theorem 2.11 implies that $\mathcal{H}_{\chi}=\operatorname{End}_{G}\left(\operatorname{ind}_{H}^{G} \chi^{-1}\right)$ decomposes as a direct sum of endomorphism rings over the different blocks, if it is commutative then in particular so are its projections to each cuspidal block. This allows us to study the relation between commutativity of $\mathcal{H}_{\chi}$ and the twisted Gelfand property of $(G, H)$ on each block separately. Since cuspidal blocks have a relatively simple description, they are easier to analyze than a general block.

Let $\rho$ be an irreducible cuspidal representation of $G$. Recall that in Section 2.2 we defined,

$$
Z:=Z\left(R_{\rho}\right) \subseteq B:=\mathcal{O}\left(\mathfrak{X}_{G}\right) \subseteq R_{\rho}:=\operatorname{End}_{G}\left(\operatorname{ind}_{G_{0}}^{G}\left(\left.\rho\right|_{G_{0}}\right)\right),
$$

and that furthermore we had $H_{\rho, V}:=\operatorname{Hom}_{G}\left(\operatorname{ind}_{G_{0}}^{G}\left(\left.\rho\right|_{G_{0}}\right), V\right)$ for a $G$-representation $V$.

Proposition 5.1. Let $Z_{\rho} \subset B \subset R_{\rho}$ be as above. If $N$ is an $R_{\rho}$-module then $\operatorname{End}_{R_{\rho}} N$ is commutative $\Longleftrightarrow \operatorname{End}_{B \otimes_{Z_{\rho}} R_{\rho}} B \otimes_{Z_{\rho}} N$ is commutative.

We prove Proposition 5.1 in two steps (Lemma 5.2 and Proposition 5.3 below).

Lemma 5.2. Let $Z \subseteq B$ be commutative rings such that $\operatorname{Spec}(B) \rightarrow \operatorname{Spec}(Z)$ is faithfully flat, and let $R$ be a $B$-algebra. Then $R$ is commutative $\Longleftrightarrow B \otimes_{Z} R$ is commutative.

Proof. Assume $B \otimes_{Z} R$ is commutative, and consider the ideal $I=R\left(r_{1} r_{2}-r_{2} r_{1}\right)$ where $r_{1}, r_{2} \in$ $R$. Since $B \otimes_{Z} I=0$, we get $r_{1} r_{2}-r_{2} r_{1}=0$ by faithfully flatness.

Since $B$ is commutative, the other direction is clear.

Proposition 5.3 (A non-commutative variant of [Eis95, Proposition 2.10]). Let $Z$ be a commutative ring, let $B$ be a flat commutative $Z$-algebra, and let $R$ be a Noetherian $Z$-algebra. Assume we are given $R$-modules $N_{1}$ and $N_{2}$ such that $N_{1}$ is finitely presented. Then the natural map

$$
\begin{gathered}
\kappa: B \otimes_{Z} \operatorname{Hom}_{R}\left(N_{1}, N_{2}\right) \rightarrow \operatorname{Hom}_{B \otimes_{Z} R}\left(B \otimes_{Z} N_{1}, B \otimes_{Z} N_{2}\right), \\
\kappa\left(b \otimes_{Z} \varphi\right)\left(b^{\prime} \otimes_{Z} n\right)=b b^{\prime} \otimes_{Z} \varphi(n)
\end{gathered}
$$

is an isomorphism.

(1) If furthermore $N_{1} \simeq N_{2}$, then $\kappa$ is an isomorphism of algebras.

(2) In particular, if $Z=R$ and $B=S^{-1} Z$ where $S \subset Z$ is multiplicatively closed then flatness of $S^{-1} Z$ over $Z$ implies $\kappa$ induces an isomorphism

$$
\kappa: S^{-1} \operatorname{Hom}_{Z}\left(N_{1}, N_{2}\right) \stackrel{\sim}{\rightarrow} \operatorname{Hom}_{S^{-1} Z}\left(S^{-1} N_{1}, S^{-1} N_{2}\right) .
$$

Proof. If $N_{1} \simeq N_{2}$, this is clearly a map of rings.

We prove $\kappa$ is an isomorphism in two steps. Assume $N_{1} \simeq R^{m}$ is a free $R$-module. We have

$$
\begin{gathered}
B \otimes_{Z} \operatorname{Hom}_{R}\left(N_{1}, N_{2}\right)=B \otimes_{Z} \operatorname{Hom}_{R}\left(R^{m}, N_{2}\right) \simeq\left(B \otimes_{Z} N_{2}\right)^{m} \\
\operatorname{Hom}_{B \otimes_{Z} R}\left(B \otimes_{Z} N_{1}, B \otimes_{Z} N_{2}\right) \simeq \operatorname{Hom}_{B \otimes_{Z} R}\left(\left(B \otimes_{Z} R\right)^{m}, B \otimes_{Z} N_{2}\right) \simeq\left(B \otimes_{Z} N_{2}\right)^{m} .
\end{gathered}
$$

In this presentation, the map $\kappa$ is given on generators by $\kappa\left((b \otimes n) e_{j}\right)=(b \otimes n) e_{j}$, so it is an isomorphism.

For the general case, write a free resolution $P^{\bullet}$ of $N_{1}$ :

$$
P^{\bullet} \rightarrow N_{1} \rightarrow 0=\cdots \rightarrow \underset{16}{P^{2} \rightarrow P^{1} \rightarrow P^{0} \rightarrow N_{1} \rightarrow 0 .}
$$


Since $B$ is flat over $Z$, tensoring preserves cohomologies:

$$
B \otimes_{Z} \operatorname{Ext}_{R}^{i}\left(N_{1}, N_{2}\right)=B \otimes_{Z} H^{i}\left(\operatorname{Hom}_{R}\left(P^{\bullet}, N_{2}\right)\right)=H^{i}\left(B \otimes_{Z} \operatorname{Hom}_{R}\left(P^{\bullet}, N_{2}\right)\right) .
$$

Consider the chain map $\kappa^{\bullet}: B \otimes_{Z} \operatorname{Hom}_{R}\left(P^{\bullet}, N_{2}\right) \rightarrow \operatorname{Hom}_{B \otimes_{Z} R}\left(B \otimes_{Z} P^{\bullet}, B \otimes_{Z} N_{2}\right)$. Each $P^{i}$ is free, so by Step 1 the maps $\kappa^{i}: B \otimes_{Z} \operatorname{Hom}_{R}\left(P^{i}, N_{2}\right) \rightarrow \operatorname{Hom}_{B \otimes_{Z} R}\left(B \otimes_{Z} P^{i}, B \otimes_{Z} N_{2}\right)$ are isomorphisms and thus so is $\kappa^{\bullet}$. Since $B \otimes_{Z}(-)$ is exact $B \otimes_{Z} P^{\bullet}$ is a free resolution of $B \otimes_{Z} N_{1}$. We get,

$$
\begin{aligned}
H^{i}\left(B \otimes_{Z} \operatorname{Hom}_{R}\left(P^{\bullet}, N_{2}\right)\right) & \simeq H^{i}\left(\operatorname{Hom}_{B \otimes_{Z} R}\left(B \otimes_{Z} P^{\bullet}, B \otimes_{Z} N_{2}\right)\right) \\
& =\operatorname{Ext}_{B \otimes_{Z} R}^{i}\left(B \otimes_{Z} N_{1}, B \otimes_{Z} N_{2}\right) .
\end{aligned}
$$

In particular, setting $i=0$ we see that $\kappa^{0}$ induces the desired isomorphism $\kappa$.

Proof of Proposition 5.1. By Theorem 2.12 the map $\operatorname{Spec}(B) \rightarrow \operatorname{Spec}(Z)$ is surjective and étale, so it is faithfully flat. By Lemma 5.2 we have $\operatorname{End}_{R_{\rho}}(N)$ is commutative $\Longleftrightarrow B \otimes_{Z_{\rho}} \operatorname{End}_{R_{\rho}}(N)$ is commutative. Using Proposition 5.3 we get $B \otimes_{Z_{\rho}} \operatorname{End}_{R_{\rho}} N \simeq \operatorname{End}_{B \otimes_{Z_{\rho}} R_{\rho}}\left(B \otimes_{Z_{\rho}} N\right)$ and the claim follows.

For the proofs of the next two statements set $M=\operatorname{ind}_{H}^{G} \chi^{-1} \delta$.

Proposition 5.4. $\operatorname{End}_{R_{\rho}}\left(H_{\rho, \operatorname{ind}_{H}^{G} \chi^{-1} \delta}\right)$ is commutative $\Longleftrightarrow \operatorname{End}_{B}\left(\left.H_{\rho, \operatorname{ind}_{H}^{G} \chi^{-1} \delta}\right|_{B}\right)$ is commutative.

Proof. Assume $\operatorname{End}_{R_{\rho}}\left(H_{\rho, M}\right)$ is commutative. By Theorem 2.12 we have,

$$
\left(B \otimes_{Z_{\rho}} Z_{\rho} \subseteq B \otimes_{Z_{\rho}} B \subseteq B \otimes_{Z_{\rho}} R_{\rho}\right) \simeq\left(B \cdot \mathrm{Id} \subseteq \operatorname{diag}(B) \subseteq \mathrm{M}_{n}(B)\right) .
$$

Set $N=B \otimes_{Z_{\rho}} H_{\rho, M}$. It is an $\mathrm{M}_{n}(B)$-module, and by Morita equivalence we have $N \simeq \tilde{N}^{n}$ where $\tilde{N}$ is a $B$-module and $\operatorname{End}_{B}(\tilde{N}) \simeq \operatorname{End}_{\mathrm{M}_{n}(B)}(N)$.

Since by Proposition 5.1 the algebra $\operatorname{End}_{B \otimes_{Z_{\rho}} R_{\rho}}(N)$ is commutative, we conclude that the algebra $\operatorname{End}_{B \otimes Z_{\rho} B}\left(B \otimes_{Z_{\rho}} H_{\rho, M}\right)$ is commutative as well:

$$
\operatorname{End}_{B \otimes_{Z_{\rho}} B}\left(B \otimes Z_{\rho} H_{\rho, M}\right)=\operatorname{End}_{B^{n}}(N)=\operatorname{End}_{B^{n}}\left(\tilde{N}^{n}\right) \simeq\left(\operatorname{End}_{B}(\tilde{N})\right)^{n} .
$$

By applying Proposition 5.1 once again, we see that $\operatorname{End}_{B}\left(\left.H_{\rho, M}\right|_{B}\right)$ is commutative.

The converse is clear since $B \subset R_{\rho}$ implies $\operatorname{End}_{R_{\rho}}\left(H_{\rho, M}\right) \subseteq \operatorname{End}_{B}\left(\left.H_{\rho, M}\right|_{B}\right)$.

Recall $(G, H):=(\mathbf{G}(F), \mathbf{H}(F))$ is an $F$-spherical pair if $|H \backslash G / P|$ is finite for every $P=\mathbf{P}(F)$ where $\mathbf{P}$ is a parabolic of $\mathbf{G}$. Theorem $\mathbf{C}$ now clearly follows from Theorem 5.5 by ranging over all irreducible cuspidal representations $\rho$.

Theorem 5.5. Let $(G, H)$ be an F-spherical pair, assume $d_{H, \chi \delta}(\rho)<\infty$ and denote by $\left(\mathcal{H}_{\chi}\right)_{\rho}:=$ $\operatorname{End}_{R_{\rho}}\left(H_{\rho, \text { ind }_{H}^{G} \chi^{-1} \delta}\right)$ the projection of $\mathcal{H}_{\chi}$ to the block $\mathcal{B}_{\rho}$. Then

$\left(\mathcal{H}_{\chi}\right)_{\rho}$ is commutative $\Longleftrightarrow d_{H, \chi \delta}\left(\rho^{\prime}\right) \leq 1$ for every irreducible representation $\rho^{\prime} \in \mathcal{B}_{\rho}$.

Proof. Assume $H_{\rho, M} \neq 0$, as otherwise we are done. Using Theorem 2.14(2) we have $H_{\rho, M}=$ $i_{*}(\mathcal{F})$ where $i: \mathfrak{X}^{\prime} \subset \mathfrak{X}_{G}$ is smooth and $\mathcal{F}$ is locally free. Since $\mathcal{F}$ is locally free, $\operatorname{supp}(\mathcal{F})$ is a union of irreducibility components of $\mathfrak{X}^{\prime}$, so we can assume $\mathfrak{X}^{\prime}$ is the vanishing set of $I=\operatorname{Ann}_{B}\left(H_{\rho, M}\right)$, the annihilator of $H_{\rho, M}$ in $B$. 
We get that $H_{\rho, M}$ is locally free as a $B / I$-module, so there exist generators $f_{1}, \ldots, f_{n} \in B / I$ such that $f_{i}^{-1} H_{\rho, M} \simeq\left(f_{i}^{-1}(B / I)\right)^{k_{i}}$ for some $k_{i} \in \mathbb{N}$ and $\bigcup_{i=1}^{n} D\left(f_{i}\right)=\operatorname{Spec}(B / I)$.

If $\operatorname{End}_{R_{\rho}}\left(H_{\rho, M}\right)$ is commutative, then by Proposition 5.4 so is $\operatorname{End}_{B / I}\left(H_{\rho, M}\right)$. By Corollary B.4.6 of [AGS15, Appendix B] (see also AAG12]), $H_{\rho, M}$ is finitely generated over $B$, so it is a finitely presented $B / I$ module ( $B / I$ is Noetherian). Now, Proposition 5.3(2) implies End $_{f_{i}^{-1} B / I}\left(f_{i}^{-1} H_{\rho, M}\right) \simeq f_{i}^{-1} \operatorname{End}_{B / I}\left(H_{\rho, M}\right)$ is commutative. We conclude that for each $i$ we have $f_{i}^{-1} H_{\rho, M} \simeq f_{i}^{-1}(B / I)$.

By Theorem 2.14 $(1), \operatorname{Hom}_{G}(M, \rho \otimes \psi) \simeq \operatorname{Hom}_{\mathcal{O}_{\mathfrak{x}_{G}}}\left(H_{\rho, M}, \delta_{\psi}\right)$, where $\delta_{\psi}$ is the skyscraper sheaf at $\psi \in \mathfrak{X}_{G}$ with ring $\mathcal{O}_{\mathfrak{X}_{G}, \psi} / \mathfrak{m}_{\psi}$. Let $\left(H_{\rho, M}\right)_{\psi}$ be the stalk of $H_{\rho, M}$ at $\psi$. We have,

$$
\operatorname{Hom}_{\mathcal{O}_{\mathfrak{X}_{G}}}\left(H_{\rho, M}, \delta_{\psi}\right) \simeq \operatorname{Hom}_{\mathcal{O}_{\mathfrak{X}_{G}, \psi}}\left(\left(H_{\rho, M}\right)_{\psi}, \mathcal{O}_{\mathfrak{X}_{G}, \psi} / \mathfrak{m}_{\psi}\right) .
$$

Since $H_{\rho, M}$ is locally free of rank 1 over $\mathfrak{X}^{\prime}$ (and vanishes over the complement of $\mathfrak{X}^{\prime}$ ), in particular $\left(H_{\rho, M}\right)_{\psi} \simeq\left(\mathcal{O}_{\mathfrak{X}_{G}, \psi} / \mathfrak{m}_{\psi}\right)^{k}$ where $k \leq 1$. We get $d_{H, \chi \delta}(\rho \otimes \psi)=\operatorname{dim}_{\mathbb{C}} \operatorname{Hom}_{G}(M, \rho \otimes \psi) \leq$ 1:

$\operatorname{dim}_{\mathbb{C}} \operatorname{Hom}_{\mathcal{O}_{\mathfrak{X}_{G}}}\left(H_{\rho, M}, \delta_{\psi}\right)=\operatorname{dim}_{\mathbb{C}} \operatorname{Hom}_{\mathcal{O}_{\mathfrak{X}_{G}, \psi}}\left(\left(H_{\rho, M}\right)_{\psi}, \mathcal{O}_{\mathfrak{X}_{G}, \psi} / \mathfrak{m}_{\psi}\right)=\operatorname{dim}_{\mathbb{C}} \operatorname{Hom}_{\mathcal{O}_{\mathfrak{X}_{G}, \psi}}\left(\mathcal{O}_{\mathfrak{X}_{G}, \psi}^{k}, \mathcal{O}_{\mathfrak{X}_{G}, \psi} / \mathfrak{m}_{\psi}\right) \leq 1$.

Since every irreducible $\rho^{\prime} \in \mathcal{B}_{\rho}$ is of the form $\rho^{\prime} \simeq \rho \otimes \psi$ for some $\psi \in \mathfrak{X}_{G}$, we are done.

Conversely, we get the following for every $\psi \in \mathfrak{X}_{G}$ :

$$
\operatorname{dim}_{\mathbb{C}} \operatorname{Hom}_{\mathcal{O}_{\mathfrak{X}_{G}, \psi}}\left(\left(H_{\rho, M}\right)_{\psi}, \mathcal{O}_{\mathfrak{X}_{G}, \psi} / \mathfrak{m}_{\psi}\right)=\operatorname{dim}_{\mathbb{C}} \operatorname{Hom}_{\mathcal{O}_{\mathfrak{X}_{G}}}\left(H_{\rho, M}, \delta_{\psi}\right)=d_{H, \chi \delta}(\rho \otimes \psi) \leq 1 .
$$

Recalling the locally freeness of $H_{\rho, M}$ over $\mathfrak{X}^{\prime}$, the rank of every $f_{i}^{-1} H_{\rho, M}$ is at most 1 . It follows every $f_{i}^{-1} \operatorname{End}_{B / I}\left(H_{\rho, M}\right) \simeq \operatorname{End}_{f_{i}^{-1}(B / I)}\left(f_{i}^{-1} H_{\rho, M}\right)$ is commutative. Since $\left\{f_{i}\right\}_{i=1}^{n}$ generate $B / I$, the map

$$
\operatorname{End}_{B / I}\left(H_{\rho, M}\right) \rightarrow f_{1}^{-1} \operatorname{End}_{B / I}\left(H_{\rho, M}\right) \times \ldots \times f_{n}^{-1} \operatorname{End}_{B / I}\left(H_{\rho, M}\right)
$$

is injective, and we get that $\operatorname{End}_{B / I}\left(H_{\rho, M}\right)$ is commutative. Proposition 5.4 finishes the proof.

\section{A tempered $\chi$-Gelfand property implies $\mathcal{H}_{\chi}$ is Commutative}

Let $H \leq G$ be l.c.t.d groups as in Section 1.6. Throughout this section, we assume $G$ is second countable of type I (see Section 2.4) and that $\chi$ is a unitary character. Let $W(\chi):=$ $L^{2}\left(H \backslash G, \chi^{-1} \delta\right)$ be the completion of the unitarizable representation $\operatorname{ind}_{H}^{G} \chi^{-1} \delta$, and recall $\rho \in$ $\operatorname{Irr}(G)$ is said to be $\left(H \backslash G, \chi^{-1}\right)$-tempered if it is included in the support of the Plancherel measure of $W(\chi)$ (see Section 2.4).

Definition 6.1. We say that $(G, H)$ is an $\left(H \backslash G, \chi^{-1}\right)$-tempered $\chi$-Gelfand pair if $d_{H, \chi \delta} \leq 1$ for every $\left(H \backslash G, \chi^{-1}\right)$-tempered $\rho \in \operatorname{Irr}(G)$.

In this section we prove the following theorem.

Theorem 6.2. Let $(G, H)$ be an $\left(H \backslash G, \chi^{-1}\right)$-tempered $\chi$-Gelfand pair, then $\mathcal{H}_{\chi}$ is commutative.

Given the Hecke algebra $A(G)$ of locally constant, compactly supported measures on $G$, a standard result $([\mathrm{BR}$, Separation Lemma]) asserts that for any $0 \neq a \in A(G)$ there exists $\rho \in \operatorname{Irr}(G)$ such that $\rho(h) \neq 0$. One then says that $A(G)$ is separated by the set of its irreducible representations. We now formulate and prove an analogous statement for $\operatorname{ind}_{H}^{G} \chi^{-1} \delta$ from which, after an additional step, Theorem 6.2 will follow. 
Definition 6.3. Let $M$ be a $G$-representation, and let $\operatorname{rad}(M)$ be the intersection of its maximal sub-representations.

(1) We say that $M$ is semi-primitive or separated if $\operatorname{rad}(M)=\{0\}$.

(2) We say that $M$ is separated by a set $\left\{\left(M_{\gamma}, p_{\gamma}: M \rightarrow M_{\gamma}\right)\right\}_{\gamma \in I}$ if every $M_{\gamma}$ is an irreducible $G$-representation and for every $m \in M$ there exists $\gamma \in I$ such $p_{\gamma}(m) \neq 0$.

Let $\nu_{W(\chi)}$ be the Plancherel measure of $W(\chi):=L^{2}\left(H \backslash G, \chi^{-1} \delta\right)$, denote by $\rho^{\text {sm }}$ the smooth vectors of $\rho$ and set $S_{\nu_{W(\chi)}}:=\left\{\rho_{\gamma}^{\mathrm{sm}}: \exists \alpha_{\gamma}: L^{2}\left(H \backslash G, \chi^{-1} \delta\right) \rightarrow \rho_{\gamma}\right.$ and $\left.\left(\rho_{\gamma}, \alpha_{\gamma}\right) \in \operatorname{supp}\left(\nu_{W(\chi)}\right)\right\}$.

Proposition 6.4. The module $\operatorname{ind}_{H}^{G} \chi^{-1} \delta$ is separated by the set $\left\{\left(\rho_{\gamma}^{\mathrm{sm}}, \alpha_{\gamma}\right)\right\}_{\left(\rho_{\gamma}, \alpha_{\gamma}\right) \in \operatorname{supp}\left(\nu_{W(\chi)}\right)}$.

Proof. Consider the central decomposition of $W(\chi)$

$$
W(\chi):=L^{2}\left(H \backslash G, \chi^{-1} \delta\right)=\int_{\widehat{G}}^{\oplus} m_{\nu_{W(\chi)}}(\rho) \rho d \nu_{W(\chi)}(\rho) .
$$

By Proposition 2.17, the embedding $\alpha: \operatorname{ind}_{H}^{G} \chi^{-1} \delta \rightarrow W(\chi)$ is pointwise defined. This implies we have a family of $G$-maps $\left\{\alpha_{\gamma}: \operatorname{ind}_{H}^{G} \chi^{-1} \delta \rightarrow \rho_{\gamma}^{\text {sm }}\right\}_{\gamma \in I}$ where $\rho_{\gamma} \in \widehat{G}$, such that for every $f \in \operatorname{ind}_{H}^{G} \chi^{-1} \delta$ we have $\alpha(f)(\gamma)=\alpha_{\gamma}(f)$. In particular $\left\{\left(\rho_{\gamma}, \alpha_{\gamma}\right): \alpha_{\gamma}(f) \neq 0\right\}$ has positive measure for every $f \in \operatorname{ind}_{H}^{G} \chi^{-1} \delta$.

The following is the final ingredient needed to prove Theorem 6.2.

Proposition 6.5. The map $\phi_{\chi}: \mathcal{H}_{\chi} \rightarrow \prod_{\rho \in S_{\nu_{W}(\chi)}} \operatorname{End}_{\mathbb{C}}\left(\operatorname{Hom}_{G}\left(\operatorname{ind}_{H}^{G} \chi^{-1} \delta, \rho\right)\right)$ via

$$
\varphi \mapsto \phi_{\chi}(\varphi)\left(\left(\alpha_{\rho}\right)_{\rho \in S_{\nu_{W(\chi)}}}\right)=\left(\alpha_{\rho} \circ \varphi\right)_{\rho \in S_{\nu_{W}(\chi)}}
$$

is an injective map of rings.

Proof. This is clearly a map of rings. Given a non-zero $\varphi \in \mathcal{H}_{\chi}$, there exists $f$ such that $\varphi(f) \neq 0$, and by the previous proposition there exist an irreducible representation $\rho_{\gamma} \in S_{\nu_{W(\chi)}}$ and $\alpha_{\gamma}: \operatorname{ind}_{H}^{G} \chi^{-1} \delta \rightarrow \rho_{\gamma}$ such that $\alpha_{\gamma}(\varphi(f)) \neq 0$, as required.

Theorem 6.2 now follows immediately:

Proof of Theorem 6.2. If $(G, H)$ is an $\left(H \backslash G, \chi^{-1}\right)$-tempered $\chi$-Gelfand pair, then

$$
\prod_{\rho \in S_{\nu_{W}(\chi)}} \operatorname{End}_{\mathbb{C}}\left(\operatorname{Hom}_{G}\left(\operatorname{ind}_{H}^{G} \chi^{-1} \delta, \rho\right)\right)
$$

is a product of one dimensional algebras. Since $\phi$ is injective, $\mathcal{H}_{\chi}$ must be commutative.

\section{Appendix A. A general proof of the Gelfand-Kazhdan Criterion}

In this appendix we prove Theorem 1.6] in the case of a locally compact, totally disconnected, second countable topological group $G$ with respect to a character $\chi$ of $H \leq G$ where we do not assume either $G$ or $H$ are unimodular. Let $A(G)$ be the algebra of smooth, compactly supported measures on $G$, and let $C^{-\infty}(G)$ be its dual space, the space of generalized functions on $G$.

Theorem A.1 (Gelfand-Kazhdan criterion). Assume there exists an anti-involution $\sigma: G \rightarrow G$ such that $\sigma(H)=H$ and $\sigma(\xi)=\xi$ for every generalized function $\xi \in C^{-\infty}(G)^{\left(H, \delta_{G} \chi^{-1}\right) \times\left(H, \delta_{G} \chi^{\sigma}\right)}$. Then we have

$$
\operatorname{dim}_{\mathbb{C}} \operatorname{Hom}_{H}(\rho, \chi) \cdot \operatorname{dim}_{\mathbb{C}} \operatorname{Hom}_{H}\left(\tilde{\rho}, \chi^{\mu}\right) \leq 1,
$$


for every smooth irreducible admissible representation $\rho$ of $G$, where $\mu(g)=\sigma(g)^{-1}$.

Proof. We essentially follow Gro91, Proposition 4.2] (see also [Pra90, Lemma 4.2]), using Lemma 3.7 to replace a few arguments.

Let $\rho$ be an irreducible representation of $G$ and let $l: \rho \rightarrow \mathbb{C}_{\chi}$ and $m: \tilde{\rho} \rightarrow \mathbb{C}_{\chi^{\mu}}$ be non-zero $H$-quasi-invariant linear functionals. Choose a right invariant Haar measure $d_{r} g$ on $G$. Then the functionals above give surjective $G$-linear maps $F_{l}: A(G) \rightarrow \tilde{\rho}$ and $F_{m}: A(G) \rightarrow \tilde{\tilde{\rho}} \simeq \rho$ defined by

$$
F_{l}(f)=\int_{G}\left\langle\rho^{*}(g) l,-\right\rangle d f(g)=\rho^{*}(f)(l) \in \tilde{\rho}, \quad F_{m}(f)=\int_{G}\left\langle\tilde{\rho}^{*}(g) m,-\right\rangle d f(g)=\tilde{\rho}^{*}(f)(m) \in \rho
$$

for $f \in A(G)$. Since $\rho$ and $\tilde{\rho}$ are irreducible, the linear maps $F_{l}$ and $F_{m}$ are determined (up to a scalar) by their kernels (note we use here Schur's lemma). Composing with the $G$-invariant bilinear form $\langle-,-\rangle: \rho \times \tilde{\rho} \rightarrow \mathbb{C}$ we obtain a linear map, $B\left(f_{1}, f_{2}\right)=\left\langle F_{m}\left(f_{1}\right), F_{l}\left(f_{2}\right)\right\rangle$

$$
B: A(G) \otimes A(G) \rightarrow \rho \otimes \tilde{\rho} \rightarrow \mathbb{C} .
$$

We now claim $B$ may be viewed as a generalized function on $G \times G$ which is left equivariant under $\left(H, \delta_{G} \chi^{\sigma}\right) \times\left(H, \delta_{G} \chi^{-1}\right)$ and right invariant under the diagonal action of $G$. We calculate,

$$
\left\langle L_{\left(h_{1}, h_{2}\right)} B, f_{1} \otimes f_{2}\right\rangle=B\left(L_{h_{1}^{-1}} f_{1}, L_{h_{2}^{-1}} f_{2}\right)=\left\langle F_{m}\left(L_{h_{1}^{-1}} f_{1}\right), F_{l}\left(L_{h_{2}^{-1}} f_{2}\right)\right\rangle .
$$

Now, recall that $m$ is $\left(H, \chi^{\mu}\right)$-equivariant,

$$
\begin{aligned}
F_{m}\left(L_{h_{1}^{-1}} f_{1}\right)(\tilde{v}) & =\int_{G}\langle m, \tilde{\rho}(g) \tilde{v}\rangle d L_{h_{1}^{-1}} f_{1}(g)=\int_{G} \delta_{G}\left(h_{1}\right)\left\langle m, \tilde{\rho}\left(h_{1}^{-1}\right) \tilde{\rho}(g) \tilde{v}\right\rangle d f_{1}(g) \\
& =\delta_{G}\left(h_{1}\right) \int_{G} \chi^{\mu}\left(h_{1}^{-1}\right)\langle m, \tilde{\rho}(g) \tilde{v}\rangle d f_{1}(g)=\delta_{G}\left(h_{1}\right) \chi^{\sigma}\left(h_{1}\right) F_{m}\left(f_{1}\right)(\tilde{v}) .
\end{aligned}
$$

Similarly, $F_{l}\left(L_{h_{2}^{-1}} f_{2}\right)=\delta_{G}\left(h_{2}\right) \chi^{-1}\left(h_{2}\right) F_{l}\left(f_{2}\right)$. Since $F_{m}$ and $F_{l}$ are $G$-linear (recall our measure is $G$-invariant on the right), $F_{m}\left(R_{g^{\prime}} f\right)=\rho\left(g^{\prime}\right)\left(F_{m}(f)\right)$, and similarly for $F_{l}$. Since our bilinear form is $G$-invariant, we get that $B$ is invariant with respect to right $\Delta G$ action:

$$
\left\langle R_{(g, g)} B, f_{1} \otimes f_{2}\right\rangle=\left\langle F_{m}\left(R_{g^{-1}} f_{1}\right), F_{l}\left(R_{g^{-1}} f_{2}\right)\right\rangle=\left\langle\tilde{\rho}(g) F_{m}\left(f_{1}\right), \rho(g) F_{l}\left(f_{2}\right)\right\rangle=\left\langle F_{m}\left(f_{1}\right), F_{l}\left(f_{2}\right)\right\rangle .
$$

Now, $B \in C^{-\infty}(G \times G)^{\left(H, \delta_{G} \chi^{-1}\right) \times\left(H, \delta_{G} \chi^{\sigma}\right) \times \Delta G}$, and by Lemma3.7the isomorphism $C^{-\infty}(G) \simeq$ $C^{-\infty}(G \times G)^{\Delta G}$ induces the involution $\left(f_{1} \otimes f_{2}\right)^{\sigma}=f_{2}^{\mu} \otimes f_{1}^{\mu}$ on $C^{-\infty}(G \times G)^{\Delta G}$. By our assumptions $B$ is invariant under $\sigma$. In particular,

$$
\left\langle F_{m}\left(f_{1}\right), F_{l}\left(f_{2}\right)\right\rangle=B\left(f_{1}, f_{2}\right)=B^{\sigma}\left(f_{1}, f_{2}\right)=B\left(f_{2}^{\mu}, f_{1}^{\mu}\right)=\left\langle F_{m}\left(f_{2}^{\mu}\right), F_{l}\left(f_{1}^{\mu}\right)\right\rangle
$$

for all $f_{1}, f_{2} \in A(G)$. This means $f_{1} \in \operatorname{ker} F_{m} \Longleftrightarrow f_{1}^{\mu} \in \operatorname{ker} F_{l}$ (we can fix $f_{1}$ and let $f_{2}$ vary), so $F_{l}$ determines the kernel of $F_{m}$, and since $\rho$ is irreducible it determines $F_{m}$ up to a scalar. Since $l$ was arbitrary, we deduce that $\operatorname{dim}_{\mathbb{C}}\left(\tilde{\rho}, \chi^{\mu}\right) \leq 1$, and $\operatorname{similarly} \operatorname{dim}_{\mathbb{C}}(\rho, \chi) \leq 1$.

\section{REFERENCES}

[AAG12] Aizenbud, A., N. Avni and D. Gourevitch, Spherical pairs over close local fields, Comment. Math. Helv. 87 (2012), no. 4, 929-962.

[AG09] Aizenbud, A. and D. Gourevitch, Generalized Harish-Chandra descent, Gelfand pairs, and an Archimedean analog of Jacquet-Rallis's theorem, Duke Math. J. 149 (2009), no. 3, 509-567, with an appendix by the authors and Eitan Sayag. 
[AGRS10] Aizenbud, A., D. Gourevitch, S. Rallis, and G. Schiffmann, Multiplicity one theorems, Ann. of Math. (2) 172 (2010), no. 2, 1407-1434.

[AGS08] Aizenbud, A., D. Gourevitch and E. Sayag, $\left(\mathrm{GL}_{n+1}(F), \mathrm{GL}_{n}(F)\right)$ is a Gelfand pair for any local field $F$, Compos. Math. 144 (2008), no. 6, 1504-1524.

[AGS15] Aizenbud, A., D. Gourevitch and E. Sayag, Z-finite distributions on p-adic groups, Adv. Math. 285 (2015), 1376-1414.

[AS20] Aizenbud, A. and E. Sayag, Homological multiplicities in representation theory of p-adic groups, Math. Z. 294 (2020), no. 1-2, 451469.

[Ber74] Bernšteĭn I. N., All reductive p-adic groups are of type I, Funkcional. Anal. i Priložen. 8 (1974), no. 2, $3-6$.

[Ber88] Bernstein J. N., On the support of Plancherel measure, J. Geom. Phys. 5 (1988), no. 4, 663-710 (1989).

[BD84] Bernstein, J. N., Le "centre" de Bernstein, Representations of reductive groups over a local field, Travaux en Cours, Hermann, Paris, 1984, Edited by P. Deligne, pp. 1-32.

[BR] Bernstein J. N. and K. Rumelhart, Lectures on p-adic groups, Unpublished, available at http://www.math.tau.ac.il/ bernstei/Publication_list/publication_texts/Bernst_Lecture_p-adic_repr.pdf

[BZ76] Bernšteĭn I. N. and A. V. Zelevinskiǔ, Representations of the group $G L(n, F)$, where $F$ is a local nonArchimedean field, Uspehi Mat. Nauk 31 (1976), no. 3(189), 5-70.

[BC95] Bost J.-B. and A. Connes, Hecke algebras, type III factors and phase transitions with spontaneous symmetry breaking in number theory, Selecta Math. (N.S.) 1 (1995), no. 3, 411-457.

[Cog04] James W. Cogdell, Lectures on L-functions, converse theorems, and functoriality for $\mathrm{GL}_{n}$, Lectures on automorphic L-functions, Fields Inst. Monogr., vol. 20, Amer. Math. Soc., Providence, RI, 2004, pp. 1-96.

[Del10] Delorme P., Constant term of smooth $H_{\psi}$-spherical functions on a reductive p-adic group, Trans. Amer. Math. Soc. 362 (2010), no. 2, 933-955.

[Dia88] Diaconis P., Group representations in probability and statistics, Institute of Mathematical Statistics Lecture Notes-Monograph Series, vol. 11, Institute of Mathematical Statistics, Hayward, CA, 1988.

[Eis95] Eisenbud D., Commutative algebra, Graduate Texts in Mathematics, vol. 150, Springer-Verlag, New York, 1995, With a view toward algebraic geometry.

[Füh05] Führ H., Abstract harmonic analysis of continuous wavelet transforms, Lecture Notes in Mathematics, vol. 1863, Springer-Verlag, Berlin, 2005.

[GK75] Gel'fand I. M. and D. A. Kajdan, Representations of the group $\operatorname{GL}(n, K)$ where $K$ is a local field, 95-118.

[GPSR97] Ginzburg D., I. Piatetski-Shapiro, and S. Rallis, L functions for the orthogonal group, Mem. Amer. Math. Soc. 128 (1997), no. 611, viii+218.

[Gro91] Gross B. H., Some applications of Gelfand pairs to number theory, Bull. Amer. Math. Soc. (N.S.) 24 (1991), no. 2, 277-301.

[Hak03] Hakim J., Supercuspidal Gelfand pairs, J. Number Theory 100 (2003), no. 2, 251-269.

[HM08] Hakim J. and F. Murnaghan, Distinguished tame supercuspidal representations, Int. Math. Res. Pap. IMRP (2008), no. 2, Art. ID rpn005, 166.

[KLQ08] Kaliszewski S., M. B. Landstad, and J. Quigg, Hecke $C^{*}$-algebras, Schlichting completions and Morita equivalence, Proc. Edinb. Math. Soc. (2) 51 (2008), no. 3, 657-695.

[KS18] Krötz B. and H. Schlichtkrull, Harmonic analysis for real spherical spaces, Acta Math. Sin. (Engl. Ser.) 34 (2018), no. 3, 341-370.

[LB16] Le Boudec, Adrien, Groups acting on trees with almost prescribed local action, Comment. Math. Helv., 91 (2016), no. 2, 253-293.

[Let82] Letac G., Les fonctions sphériques d'un couple de Gel'fand symétrique et les chaînes de Markov, Adv. in Appl. Probab. 14 (1982), no. 2, 272-294.

[Off11] Offen O., On local root numbers and distinction, J. Reine Angew. Math. 652 (2011), 165-205.

[OV96] Okounkov A. and Vershik A., A new approach to representation theory of symmetric groups, Selecta Math. (N.S.) 2 (1996), no. 4, 581-605.

[Ol'77] G. I. Ol'šanskiı̌, Classification of the irreducible representations of the automorphism groups of BruhatTits trees, Funkcional. Anal. i Priložen. 11 (1977), no. 1, 32-42, 96. 
[PR94] Platonov V. and A. Rapinchuk, Algebraic groups and number theory, Pure and Applied Mathematics, vol. 139, Academic Press, Inc., Boston, MA, 1994, Translated from the 1991 Russian original by Rachel Rowen.

[Pra90] Prasad D., Trilinear forms for representations of GL(2) and local $\epsilon$-factors, Compositio Math. 75 (1990), no. 1, 1-46.

[Rez08] Reznikov A., Rankin-Selberg without unfolding and bounds for spherical Fourier coefficients of Maass forms, J. Amer. Math. Soc. 21 (2008), no. 2, 439-477.

[RW] Reid C. D. and P. R. Wesolek, Homomorphisms into totally disconnected, locally compact groups with dense image., Forum Math. 31 (2019), no. 3, 685701.

[Ro09] Roche A., The Bernstein decomposition and the Bernstein centre, Ottawa lectures on admissible representations of reductive $p$-adic groups, Fields Inst. Monogr., vol. 26, Amer. Math. Soc., Providence, RI, 2009, pp. 3-52.

[SV17] Sakellaridis Y. and A. Venkatesh, Periods and harmonic analysis on spherical varieties, Astérisque (2017), no. 396 , viii+360.

[Sha74] Shalika J.A., The multiplicity one theorem for $\mathrm{GL}_{n}$, Ann. of Math. (2) 100 (1974), 171-193.

[SZ11] Sun B. and C.-B. Zhu, A general form of Gelfand-Kazhdan criterion, Manuscripta Math. 136 (2011), no. 1-2, 185-197.

[vD09] van Dijk, Gerrit, Introduction to harmonic analysis and generalized Gelfand pairs, De Gruyter Studies in Mathematics, vol. 36, Walter de Gruyter \& Co., Berlin, 2009.

Yotam Hendel, Faculty of Mathematics and Computer Science, The Weizmann Institute of SciENCE, Rehovot 76100, IsRAel.

E-mail address: yotam.hendel@gmail.com 